\title{
A PII-like protein regulated by bicarbonate: structural and biochemical studies of the carboxysome- associated CPII protein
}

\author{
Nicole M. Wheatley ${ }^{1}$, Kevin D. Eden ${ }^{2}$, Joanna Ngo ${ }^{2}$, Justin S. Rosinski ${ }^{2}$, \\ Michael R. Sawaya ${ }^{1}$, Duilio Cascio ${ }^{1}$, Michael Collazo ${ }^{1}$, Hamidreza Hoveida ${ }^{2}$, Wayne L. Hubbell ${ }^{2,3}$, Todd O. \\ Yeates ${ }^{1,2}$. \\ ${ }^{1}$ UCLA-DOE Institute for Genomics and Proteomics, Los Angeles, CA 90095 \\ ${ }^{2}$ Department of Chemistry and Biochemistry, University of California, Los Angeles, CA 90095 \\ ${ }^{3}$ Jules Stein Eye Institute, University of California, Los Angeles, CA 90095
}

Corresponding author:

Todd O. Yeates

611 Charles Young Dr. East

Los Angeles, CA 90095-1569

(tel) 310-206-4866

yeates@mbi.ucla.edu

Manuscript contents: 10630 words (text + references + legends); 8 figures; 1 table

Supplemental Material: 4 supplemental figures +1 supplemental table

Running Title: A PII-like protein linked to carbon concentrating mechanisms

\author{
Abbreviations and Symbols \\ BMC: bacterial microcompartment (hexameric) shell protein \\ RuBisCO: Ribulose-1,5-bisphosphate carboxylase/oxygenase \\ CPII: carboxysome-associated PII protein \\ nrPII: nitrogen regulatory PII protein \\ SDS-PAGE: sodium dodecyl sulfate polyacrylamide gel electrophoresis \\ SDSL-EPR: Site Directed Spin Labeling- Electron Paramagnetic Resonance \\ ULP1: Ubiquitin-like-specific protease 1
}

\section{Keywords}

nitrogen regulatory PII proteins, bicarbonate, allostery, carbon concentrating mechanism, carboxysome, Rubisco, nucleotide-binding, CPII, DUF2309, NADH oxidoreductase subunit 5

(C) 2016. This manuscript version is made available under the Elsevier user license http://www.elsevier.com/open-access/userlicense/1.0/ 


\section{ABSTRACT}

Autotrophic bacteria rely on various mechanisms to increase intracellular concentrations of inorganic forms of carbon (i.e. bicarbonate and $\mathrm{CO}_{2}$ ) in order to improve the efficiency with which they can be converted to organic forms. Transmembrane bicarbonate transporters and carboxysomes play key roles in accumulating bicarbonate and carbon dioxide, but other regulatory elements of carbon concentration mechanisms in bacteria are less understood. In this study, after analyzing the genomic regions around $\alpha$-type carboxysome operons, we characterize a protein that is conserved across these operons but which has not been previously studied. On the basis of a series of apo- and ligand-bound crystal structures and supporting biochemical data, we show that this protein, which we refer to as the carboxysome-associated PII protein (CPII), represents a new and distinct subfamily within the broad superfamily of previously studied PII regulatory proteins, which are generally involved in regulating nitrogen metabolism in bacteria. CPII undergoes dramatic conformational changes in response to ADP binding, and the affinity for nucleotide binding is strongly enhanced by the presence of bicarbonate. CPII therefore appears to be a unique type of PII protein that senses bicarbonate availability, consistent with its apparent genomic association with the carboxysome and its constituents.

\section{INTRODUCTION}

The enzyme RuBisCO performs a key reaction in global carbon fixation, attaching $\mathrm{CO}_{2}$ to the five-carbon ribulose bisphosphate to give two molecules of the three-carbon 3-phosphoglycerate. ${ }^{1-5}$ Owing to the limited catalytic rate and selectivity of RuBisCO for $\mathrm{CO}_{2},{ }^{6-9}$ microorganisms that use RuBisCO to fix $\mathrm{CO}_{2}$ have evolved sophisticated carbon concentrating mechanisms (CCMs) to improve the enzyme's catalytic efficiency. ${ }^{8,10-15}$ 
Diverse bacterial CCMs include elements that operate in two stages. In the first stage, bicarbonate is accumulated at high concentration in the cytosol. ${ }^{15-17}$ This can occur by inward pumping of bicarbonate ${ }^{15}$ (bicarbonate transport in symport with $\mathrm{Na}^{+}$ions ${ }^{16,18,19}$ or by coupling to ATP hydrolysis ${ }^{20,21}$ ) or by electron-transport-coupled ${ }^{22-24}$ hydration of $\mathrm{CO}_{2}$ from outside the cell to bicarbonate inside the cell. The negative charge carried by bicarbonate prevents its diffusion back across the membrane bilayer. The second stage, wherein inorganic carbon is fixed by chemical reactions into organic form, occurs within the carboxysome. Carboxysomes, graphically depicted in Figure 1A, are roughly icosahedral, intracellular microcompartments that co-encapsulate the two enzymes RuBisCO and carbonic anhydrase. ${ }^{25-28}$ The proteinaceous shell of the carboxysome is made up of a few thousand copies of a few homologous but distinct proteins from the BMC (bacterial microcompartment) family of proteins. ${ }^{29-34} \mathrm{BMC}$ shell proteins assemble into hexameric units, each of which bears a central pore for molecular transport ${ }^{30,33-35}$, and these hexamers further assemble edge-to-edge to make a complete carboxysome shell. A high concentration of cytoplasmic bicarbonate drives its net diffusion through the shell protein pores and into the carboxysome, where it is dehydrated to carbon dioxide and fixed into organic form by RuBisCO before it can escape. Over the last several years, many of the components that operate directly in the context of bacterial CCMs have been characterized. ${ }^{36-41}$ These include the structural and functional characterization of most of the constituents of the carboxysome. On the other hand, a higher-level understanding of how the carboxysome and other elements of the CCM are regulated and integrated into the cellular metabolism of bacteria is less clear.

Hints about more nuanced aspects of carboxysome and CCM function have come from analyzing the genomes of carboxysome-containing bacteria and the genomic regions around their carboxysome operons. $^{36,42,43}$ Interspersed among genes known to be more or less directly associated with carboxysome function, other conserved genes appear, which are surmised to be functionally linked to 
the carboxysome or CCMs more generally. Two types of carboxysomes ( $\alpha$ and $\beta$ ) exist in nature; their distribution falls largely along phylogenetic lines, with the $\alpha$-type occurring in chemoautotrophs and some ( $\alpha$-type) cyanobacteria and the $\beta$-type occurring exclusively in $\beta$-type cyanobacteria ${ }^{44,45}$. Across the known $\alpha$-carboxysome operons and surrounding genomic regions, a few genes coding for proteins of unknown function have been noted. ${ }^{36,42}$ One of these, CbbQ with ATPase activity, was characterized in a recent study ${ }^{46}$. Another uncharacterized protein is the focus of the present work. Proteins from this family (about 110 amino acids in length) could be classified by sequence comparison algorithms as being remote members of the PII superfamily, a group of diverse regulatory proteins, among which a number are known to regulate nitrogen metabolism.

Nitrogen regulatory PII proteins (which we refer to here as nrPIIs) are a ubiquitous class of nucleotidebinding signal transduction molecules capable of inhibiting or activating target protein activities, often through the allosteric influence of $\alpha$-ketoglutarate. Alpha-ketoglutarate is a central metabolite whose inverse relationship with the concentration of primary nitrogen metabolites (e.g. ammonia) makes it a reliable signal for nitrogen limiting conditions. ${ }^{47-50}$ Numerous X-ray crystallographic studies have illuminated the structural mechanisms by which differential ATP- and ADP-binding promotes binding and non-binding conformational states in nrPII proteins. ${ }^{51-53}$ NrPIls and many PII-like proteins bind to ATP, ADP and $\alpha$-ketoglutarate ${ }^{54,55}$, though some exceptions have been reported; a PII-like protein from Haloferax binds additionally to $\mathrm{AMP}^{56}$, and a Bacillus PII-like protein binds cyclic-di-AMP and not ATP or $A D P^{57}$. Alpha-ketoglutarate exerts its allosteric regulatory effects via interaction with $\mathrm{Mg}^{2+}$-nucleotide bound $\mathrm{nrPll}$, thereby influencing its conformation and the interactions it makes with other proteins. NrPII proteins are known to modulate the activities of a variety of primary nitrogen metabolic transcription factors, enzymes and transporters. Examples of established nrPll-regulated activities include glutamine synthesis ${ }^{58,59}$, ammonium transport ${ }^{60}$ and nitrogen fixation ${ }^{61,62}$. A study in 
Synechocystis PCC 6803 also suggested a connection between a nrPII protein and nitrate transport; phosphorylation of nrPII was affected by growth on nitrate ${ }^{63}$. NrPII proteins are implicated in the regulation of primary carbon metabolism, as well. In land plants and bacteria, a central carbon metabolic enzyme acetyl-CoA carboxylase is directly regulated by nrPIIs ${ }^{64,65}$. In cyanobacteria, nrPIls are thought to regulate nitrogen assimilation in a carbon-dependent manner ${ }^{63,66}$. In an $\alpha$-cyanobacteria species lacking nitrate/nitrite uptake activities (thus relying on ammonia as a nitrogen source), a role for nrPII in regulating bicarbonate uptake has been proposed but not experimentally demonstrated ${ }^{67}$. Additionally, the distantly related PII-type protein SbtB from $\beta$-cyanobacteria binds directly to and inhibits the activity of sodium-bicarbonate transporters when those two proteins are expressed together in a recombinant $E$. coli system. ${ }^{68}$

In the work presented here, we provide the first structural and biochemical characterization of a unique type of PIl protein whose gene occurs in a conserved fashion across $\alpha$-type carboxysome operons in chemoautotrophic bacteria. We show that its three-dimensional structure and nucleotide binding properties are affected dramatically by bicarbonate binding, thereby tying its activities to carboxysome function and the regulation of one-carbon metabolism in autotrophic bacteria.

\section{RESULTS}

\section{Conservation of a PII-like Gene in $\alpha$-Carboxysome Operons}

An analysis of genomic sequence data shows that a protein with distant similarity to nrPII proteins is associated with $\alpha$-type carboxysome operons in various lithoautotrophic bacteria as a member of a novel four-member gene cluster (Fig. 1B). Two of the four members are predicted to be integral transmembrane proteins. The first is often annotated as 'NADH-ubiquinone oxidoreductase chain 5

homolog' (NDH-5), referring to the outer-most proton-pumping subunit of respiratory Complex I. ${ }^{69,70}$ 
Interestingly, homologs of this membrane protein are also encoded near cyanobacterial $\beta$-carboxysome genes and have been shown to be involved in $\mathrm{CO}_{2}$ concentrating mechanisms in those organisms. ${ }^{22-24}$ The second, a $~ 500$ amino-acid low-sequence-complexity transmembrane protein, does not contain recognizable domains or sequence motifs. The third member, predicted to be soluble, belongs to the DUF 2309 / PF10070 family. The DUF2309 family comprises large proteins of unknown function with no homologous protein structures available in the Protein Data Bank. The NDH-5/PII four member cluster described here is one of several gene clusters that contain NDH-5 and DUF2309. The NDH-5 and DUF2309 proteins are found genetically coupled as an operon throughout the domain Bacteria and within the class Halobacteria of the Euryarchaeota. According to the ELMB database, there are $>5000$ occurrences of the NDH-5/DUF2309 pair, most of which are distributed among 11 bacterial phyla, especially concentrated among the Firmicutes (Gram positive) and Proteobacteria (Gram negative). Furthermore, to our knowledge DUF2309 is always accompanied by an NDH-5 homolog. One of the most common NDH-5/DUF2309-based clusters contains a single third gene annotated as a 'hydrogenperoxide inducible genes activator' or more generically as a 'LysR transcriptional regulator'. Based on sequence comparison methods (see Methods), the fourth member of the novel four-gene cluster reveals remote but statistically detectable homology to the ubiquitous PII signal-transducer proteins. An analysis of these carboxysome-associated PII-like proteins reveals divergent PII-like motifs (B-loop and Cloop) thought to be critical for nitrogen regulatory PII activity ${ }^{71-74}$, suggesting a distinct or specialized function. Estimates of evolutionary divergence place the carboxysome-associated PII-like proteins in a distinct clade (Fig. 1C). We therefore introduce the name CPII for these specialized carboxysomeassociated PII proteins, which have not been studied previously.

\section{Crystal Structure of Apo-CPII}

In order to characterize a CPII protein in detail, we solved a series of crystal structures of CPII from 
Thiomonas intermedia strain K12 (Genbank accession ADG29527, locus tag Tint_0114). The overall three-dimensional structure of CPII (Fig. 2) is similar to structures reported for well-studied nrPII proteins and to a structure deposited in the PDB (2CZ4) for a PII-like protein from Thermus thermophilus. ${ }^{74-78}$ PII proteins are homotrimeric with nucleotide binding sites at three identical subunit interfaces. The CPII secondary structure consists of two consecutive but interdigitated $\beta-\alpha-\beta$ motifs connected by a flexible loop that comprises the so-called "T-loop" . The two consecutive $\beta$ - $\alpha$ - $\beta$ motifs are organized so that $\beta 1$ and $\beta 4, \alpha 1$ and $\alpha 2$, and $\beta 3$ and $\beta 2$ align in antiparallel orientation (Fig. 2). In contrast to nrPII, the $\beta 2$ strand in CPII is kinked in the middle by an additional asparagine residue (Asn41) forming a $\beta$-bulge. For the purposes of comparison to nrPIls, the notations $\beta 2.1$ and $\beta 2.2$ are chosen to specify the first half of $\beta 2$ and the second half of $\beta 2$, respectively. The tertiary structure of CPII is completed as $\beta 1: \beta 4$ and $\beta 2: \beta 3$ come together through the $\beta 1: \beta 3$ antiparallel $\beta$-sheet. The resulting fourstranded $\beta$-sheet $(\beta 2: \beta 3: \beta 1: \beta 4)$ has one hydrophobic face and one more polar face. The hydrophobic face minimizes solvent exposure by abutting against $\alpha 1$ and $\alpha 2$. The other side of the four-strand $\beta$ sheet is partly exposed, facing a very small internal cavity in the trimer. The interfaces between monomers are stabilized by antiparallel $\beta$-sheet interactions involving $\beta 4-\beta 4$ and $\beta 2.1-\beta 2.2$. The structure of an apo CPII trimer is shown in two orientations in Figure 2. This description holds true for most PII and PII-like protein structures. Between CPII and canonical nrPII proteins, however, considerable deviation occurs in the coils that create the adenosine-nucleotide binding pocket, namely the B-loop, C-loop, and T-loop, as discussed below.

Our crystal structure of CPII with no ligands bound (apo-CPII, PDB ID 5D4L) reveals a flexible T-loop from Gly-46 to Phe-57 (Fig. 2). A consensus sequence of the 16 amino acid T-loop of CPII can be described (Fig. 3A) as G[SRA][HQ]G[FIM][HY][EG]GH[LV]MFNED[DEA], where residues in bold font represent the most prevalent amino acid type among alternative possibilities. Conserved residues within the T-loop 
may dictate specificity for target effectors; T-loops often mediate direct protein-protein interactions that PII proteins make with their partners, though an exception has been noted for one particular interaction between nrPII (GInZ) and DraG in Azospirillum brasilense.$^{79}$ In our apo-CPII structures, the Tloop adopts different conformations in different crystal-packing environments. If stabilized through crystal contacts, the T-loop usually lies as a coil along the length of the empty nucleotide binding pocket, with Phe-57 filling part of the adenine binding site (see PDB 5D4L chain A, 5D40 chain C). When T-loops are not stabilized by crystal contacts, electron density is weak or absent from His-48 to Met-56, indicating flexibility or multiple alternative conformations (see PDB 5D4L chain B, 5D4N chain C,). Phenylalanine-57, however, is still present in the adenine binding pocket in structures with disordered Tloops.

Other key loop regions are likewise dissimilar between CPII and well-studied nrPIls. The sequenceconserved CPII B-loop (the coil connecting $\alpha 2$ and $\beta 4$ ) is significantly shorter than the nrPII B-loop (Fig. 3B), suggesting altered nucleotide specificity. ${ }^{53,80} \mathrm{CPII}$ does not contain any Walker-A-like motif spanning its B-loop. In place of the nrPII B-loop motif [TS][GS]xxG[ED]GKhh (h being any hydrophobic residue $)^{71,81,82}$, the CPII B-loop contains a short but conserved sequence H[TS]G spanning residues 89-91, with the Ser-90 hydroxyl group pointing into the nucleotide binding cleft (Fig. 2, 3B). In all PII and PII-like sequences, the B-loop makes close, direct contacts with bound nucleotides.

A C-loop motif analogous to the one in nrPII proteins is present only in ligand-bound forms of CPII, but not in apo-CPII. The absence of a canonical C-loop in apo-CPII (Fig. 3C) suggests again that CPII behaves differently from nrPIls with respect to nucleotide binding. ${ }^{72}$ The nrPII C-loop is a small C-terminal hairpin with two conserved arginine residues that serve to interact with the phosphate groups of bound ATP. In place of the C-loop motif, the elongated $\mathrm{N}$-terminus of apo-CPII extends along the length of the C- 
terminus as an anti-parallel $\beta$-sheet. Also, instead of the conserved RxR sequence motif of the nrPII Cloop, CPII contains a conserved RxxKF sequence beginning at Arg-102. In apo-crystal structures of CPII, electron density for residues 1, 2 and 104-108 is weak or absent. Figure 3D compares nrPII $\beta 2$ strand with that of CPII, highlighting the $\beta$-bulge induced by Asn-41. Figure 3E compares the three-dimensional trimeric assemblies of nrPII and CPII proteins.

\section{Ligand-Induced Conformational Changes in CPII}

X-ray crystallography was used to visualize the effects of ligands on the structure of CPII. A series of crystal structures were determined with diffraction resolutions between $1.7-2.3 \AA$ from crystals bound by combinations of ADP, AMP, $\mathrm{MgCl}_{2}$, bicarbonate and/or its structural analog acetate. (See Table 1 and Supplemental Table 1 for details regarding crystal conditions and ligands.) Several of these structures are shown in Figure 4, highlighting the effects of ligand binding. ADP- and bicarbonate-bound structures reveal drastic conformational changes in both the C-terminus and the T-loop compared to apostructures

The CPII T-loop Phe-57 disassociates from the interior of a nucleotide binding cleft upon occupation by $A D P$, and is re-positioned to the apex of the raised T-loop with its hydrophobic phenyl ring solventexposed (Fig 4A,5). All three T-loops (Fig 4A1-A3) extend out and perpendicular to the triangular plane of the CPII trimer in the structure identified by PDB code 5D4P. This rearrangement corresponds to a movement in the T-loop of almost $20 \AA$. Similarly elevated T-loops are observed in various ADP-bound structures (Fig. 4B1 and 4C1). AMP also displaces T-loops in CPII, although they are more often disordered than perpendicularly elevated (Fig4 B2 and C2). In addition to displacing the T-loop from the nucleotide binding cleft, nucleotide binding stabilizes the C-terminus as a short $\alpha$-helix. The changes that occur in the T-loop and C-loop upon nucleotide binding are highlighted in Figure 5. The amino group of 
Lys-105 is positioned near the $\alpha$-phosphate of the nucleotide and the oxygen atom of the Gly-91 (conserved B-loop residue). Across the distinct crystal forms studied, distances between Lys-105 and the $\alpha$-phosphate vary between 2.7 and 3.0 $\AA$, while distances to the carbonyl group (of Gly-91) vary more widely from $2.8 \AA$ (PDB 5D4O) to as high as $4.7 \AA$ (PDB 5D4P).

Bicarbonate molecules bind in nooks formed by Gly-101 and Arg-102, which immediately precede the Cloop $\alpha$-helix (Fig. 4,6). The presence of bicarbonate promotes the geometry of the C-loop helix through interaction with Arg-102. Bicarbonate binding appears to jointly promote the formation of the C-loop helix. Figure 6 compares C-loops with and without bicarbonate, revealing variation in the helical geometry and re-orientation of Arg-102. Although the C-loop Lys-105 directly contacts the ADP $\alpha$ phosphate with or without bicarbonate or acetate, the helical geometry of the C-terminal segment is promoted by interaction of bicarbonate with Arg-102. Without bicarbonate or acetate bound, the side chain of Arg-102 extends away from the protein core and into the solvent.

We analyzed the dramatic ADP-dependent conformational changes in CPII in the context of conformational changes that have been observed before in well-studied nrPII proteins. In some cases, crystal structures of ADP-bound nrPlls in complex with target effectors show T-loop conformational changes as large as those found between apo-CPII to ADP-bound CPII crystal structures in this study ${ }^{51,52}$. In the case of CPII, the changes are peculiar both in terms of the articulated surface shape of the T-loops and in the hydrophobicity created by exposed T-loop residues Leu-55, Met-56 and Phe-57. The ADPbound form thus appears to be poised for interactions with other molecules, though more detailed conclusions cannot be drawn at present. The capacity for intermolecular interactions at this site is manifested by the tendency of these extended phenylalanine residues (at ADP-bound clefts) to participate in fortuitous intermolecular contacts with other trimers in some crystal forms. The C-termini 
of typical nrPII proteins are known to interact with nucleotide phosphates of ATP and ADP, but they are not known to interact with bicarbonate. However, we note that two acetate molecules were modeled in the structure of an nrPII protein GInK2 from A. fulgidus (PDB ID 3NCQ), though the biological significance of this observation (if any) is unknown.

\section{Measurement of Nucleotide Binding by Electron Paramagnetic Resonance (EPR)}

Initial attempts to measure nucleotide binding by CPII using isothermal titration calorimetry were complicated by a tendency of the protein to precipitate in the instrument, apparently as a consequence of stirring. We therefore devised an SDSL-EPR-based (Site Directed Spin Labeling- Electron Paramagnetic

Resonance) method for measuring nucleotide binding. ${ }^{83,84}$ Supplemental Figure 1 provides a detailed example of our analytical strategy. Our crystal structures (discussed below) suggested that nucleotide binding stabilizes the C-terminus of the protein in an $\alpha$-helical conformation. We therefore monitored the EPR spectrum of a nitroxide side chain (known as $\mathrm{R} 1^{83,84}$ ) introduced via an engineered C-terminal cysteine, Cys-109, with the expectation that nucleotide binding would be reflected by a reduced mobility of the R1 spin-label with corresponding changes in the EPR spectrum. The EPR spectra of CPII-C109-R1 in the presence of various concentrations of ATP, ADP or AMP were recorded. ADP and AMP produced changes reflecting a concentration-dependent decrease in R1 mobility with saturation behavior, while ATP did not appreciably change the EPR spectrum of C109R1 (Fig. 7A and Supplemental Figure 2). From the resulting binding curves (Fig. 7B) the $K_{D}$ values for ADP and AMP were measured to be 1.6 and 1.8 $\mathrm{mM}$ respectively. Those values were not substantially affected by the presence of $\mathrm{MgCl}_{2}$ nor acetate (Supplemental Figure 2).

We also investigated the possibility of identifying other effector molecules that might influence nucleotide binding by CPII. Because $\alpha$-ketoglutarate is an effector for many nrPII proteins, we tested the 
effects of that metabolite on the binding of nucleotides by CPII but found little to no effect (Supplemental Figure 2). However, we discovered instead that nucleotide binding was strongly and consistently affected by bicarbonate in a concentration-dependent manner. Figure 7B shows the binding curves of ADP to CPII-R1 in a range of concentrations of bicarbonate $(10 \mu \mathrm{M}, 5 \mathrm{mM}, 10 \mathrm{mM}, 50 \mathrm{mM}$ $\mathrm{HCO}_{3}{ }^{-}$). At high concentrations of bicarbonate, the affinity of CPII for ADP is increased by a factor of about 15 , to $230 \mathrm{uM}$. This result suggested that, at physiological concentrations of ADP, CPII might fluctuate between partially- and fully-bound forms depending on intracellular bicarbonate concentrations. To further probe this possibility, we monitored ADP binding in a solution of constant concentrations of protein and ADP (10 $\mu \mathrm{M}$ CPII-R1 and $2 \mathrm{mM}$ ADP) as a function of increasing bicarbonate (Fig. 7B and 7C). As expected, the binding affinity of CPII for ADP is strongly influenced by bicarbonate concentrations in the range of 5 to $10 \mathrm{mM}$ bicarbonate. Likewise, at a fixed physiologically relevant concentration of $2 \mathrm{mM} \mathrm{ADP}$, nucleotide binding is strongly promoted by increasing bicarbonate concentration; e.g. from about $50 \%$ saturated in the absence of bicarbonate to about $90 \%$ saturated in $10 \mathrm{mM}$ bicarbonate. Similar binding results were obtained between CPII, bicarbonate and AMP (Supplemental Figure 3). The binding data demonstrate a thermodynamic coupling between nucleotide binding and bicarbonate binding, consistent with an allosteric relationship inferred from the ligandbound crystal structures described above. Both binding events appear to stabilize similar conformational changes in the protein, especially involving the C-loop helix. Nucleotide binding stabilizes the C-terminal helix through nucleotide $\alpha$-phosphate interaction with Lys-105, while bicarbonate binding stabilizes the helix through interactions with Arg-102. An additional EPR experiment, in the absence of nucleotide, confirmed that high bicarbonate concentrations appear to stabilize the same conformation of the C-terminal helix as nucleotide binding (Supplemental Figure 4).

In order to more firmly establish that the conformational changes seen by EPR upon nucleotide addition 
are due to nucleotide binding, we performed equilibrium dialysis experiments. ADP binding experiments were performed in triplicate in the absence and presence of bicarbonate, allowing $K_{D}$ values to be calculated. The measured $K_{D}$ values were $14(+/-4) \mathrm{mM}$ in the absence of bicarbonate and $200(+/-100)$ $\mathrm{uM}$ in the presence of $50 \mathrm{mM}$ bicarbonate. The equilibrium dialysis results confirm the strong dependence of ADP affinity on the presence of bicarbonate. The numerical $K_{D}$ values are similar from EPR and equilibrium dialysis in the presence of bicarbonate, though there is an important deviation between $K_{D}$ values for the two experimental methods in the absence of bicarbonate. The nucleotide binding measurements by dialysis were made on the basis of $A_{260}$ values. This was made possible by the minimal influence from the protein at that wavelength as CPII lacks tryptophan residues, though we note that the precision of the $K_{D}$ measurements were not as high by this approach in comparison to the EPR-based measurements described above. Beyond the difference in precision of the two methods as we applied them here, this may reflect that the two experiments are reporting on distinct phenomena nucleotide binding and ordering of the C-loop - which are coupled but not identical.

\section{AMP Modeled into Various Crystal Structures}

We observed that CPII can bind both AMP and ADP using crystallographic and biochemical methods. Interestingly, several crystallization trials that aimed to co-crystallize ADP and $\mathrm{MgCl}_{2}$ with CPII (5D4N, 5DRK and 5D40, see Table 1 and Supplemental Table 1) produced crystals with electron density that could only be modeled by AMP at one of the three nucleotide binding sites, even though AMP was not added to the crystallization conditions. We are unable to determine whether or not ADP was catalytically hydrolyzed to AMP by CPII, or if some ADP non-enzymatically hydrolyzed in the crystallization conditions after several days of incubation at room temperature. We considered that the electron density modeled as AMP could instead be an ADP with a structurally disordered $\beta$-phosphate. However, in view of the high resolution of the diffraction data, the electron dense nature of the 
phosphate group, and the relative flatness of a difference electron density map in that spot, we judged this to be unlikely. Difference electron density maps (Fig. 8) revealed no density protruding from the $\alpha$ phosphate group of the modeled AMP. In a test map generated by refinement of the structure without modeled AMP, positive difference density corresponding to the $\alpha$-phosphate of AMP appears at a height of $19 \sigma$ (standard deviations above the mean), while no positive density higher than $1.5 \sigma$ appears in any position that might correspond to a $\beta$-phosphate. This eliminates the possibility that ADP occupies that nucleotide-binding cleft. Further studies are required to explain the unexpected presence of AMP in the CPII binding sites in these crystal forms.

\section{DISCUSSION}

In this study we describe a novel protein sub-family, CPII, which has diverged from a ubiquitous superfamily of regulatory PII proteins known from previous work to be involved in various facets of nitrogen metabolism. Interestingly, CPII's genomic associations indicated a connection to $\mathrm{CO}_{2}$ concentration and carbon fixation. We used structural and biochemical methods to reveal unique features of CPII compared to other PII proteins, with the binding of CPII to bicarbonate being particularly noteworthy. Those findings shed light on how CPII might operate in the cell and how its activities relate to inorganic carbon concentration in bacteria.

A series of crystal structures indicate that CPII binds to and signals through nucleotide-dependent conformational changes. We discovered that ADP binding causes major conformational changes in CPII as large as $20 \AA$, with the most dramatic changes occurring in the T-loop region (Fig. 4 and Fig. 5). The ADP-bound conformation (PDB ID 5D4P, bound to three ADP molecules) is notable for the peculiar nature of the surface it creates on one side of the trimer, where The T-loops expose phenylalanine, 
along with other hydrophobic T-loop residues, near the three-fold axis of symmetry. This creates an articulated hydrophobic surface, which we surmise corresponds to a site of interaction with a downstream signaling partner. Among well-studied nitrogen regulatory PII proteins, signaling occurs through interactions of protruding T-loop amino-acid side chains with protein partners such as the ammonium transporter $\mathrm{AmtB}^{51}$, acetyl-glutamate kinase $\mathrm{S}^{53}$ and $\mathrm{Pip} \mathrm{X}^{52}$. The cognate partner or partners of CPII will need to be defined in subsequent studies. If CPII is encoded near its target effector, as is often the case for nrPII proteins, then the NADH dehydrogenase subunit 5-like and the DUF2309 protein are plausible candidates as target effectors for CPII. Unfortunately, all attempts at heterologous production of the NDH/DUF2309/low-complexity proteins in E. coli failed, whether expressed separately or in combinations.

The ligand binding properties of CPII were explored in order to corroborate the structural data on nucleotide binding and to look for effector molecules that might show a thermodynamic coupling to nucleotide binding events, as would be expected for allosteric regulation. We found that EPR spectroscopy was a facile approach for extracting binding affinities based on the effects that ligand binding had on the mobility of a spin label attached near the presumptive binding site cleft. ${ }^{84}$ Binding affinities in the low millimolar range were found for ADP and also AMP, but no binding was detected for ATP. Most importantly, the nucleotide binding affinities increased by a factor of about 15 to give $K_{D}$ values of $230 \mathrm{uM}$ in the presence of bicarbonate, with nearly full effect exhibited in the $10 \mathrm{mM}$ concentration range for bicarbonate. This result establishes an allosteric signaling mechanism for CPII involving bicarbonate, which is particularly significant given that a key motivation of the present work was to uncover possible connections to carbon metabolism for this gene that is conserved across carboxysome and carbon concentrating operons. Estimates of intracellular concentrations of ADP and AMP range roughly between $0.2 \mathrm{mM}-1.5 \mathrm{mM}$ and $0.04-0.3 \mathrm{mM}$, respectively, ${ }^{85}$ and bicarbonate 
concentrations may exceed $50 \mathrm{mM}$ during active carbon concentration. Thus, under physiological conditions, increasing bicarbonate concentrations could shift the equilibrium position for CPII binding to ADP to a more fully bound state, with concomitant protein conformational changes driving the association of CPII with downstream effectors.

These studies on CPII provide early clues about function and mechanism, while opening up several new avenues for further exploration. If comparable to nrPIls, the relationship between nucleotide binding and CPII function is likely defined by the nucleotide's effect on the positioning of the T-loop. Our structural findings indicate that ADP binding displaces the T-loop bearing Phe-57 and also promotes conversion of the C-terminal protein segment to a helical form by way of an interaction with Lys-105. The concomitant effect on the position of Arg-102 favors binding of bicarbonate, and vice-versa. These mechanistic features tie nucleotide and bicarbonate concentrations to an altered conformation of CPII that we presume interacts with target effectors. Based on the apparent genomic association of CPII with the transmembrane NDH-5-like protein, DUF2309, and a low-complexity transmembrane protein of unknown function, together with the known regulation of ammonium transport by the interaction of well-studied nrPII proteins with the membrane transporter, AmtB, we hypothesize that CPII could exert its regulatory function through interactions with its genetically associated transmembrane proteins, which may be involved in molecular transport events that are not yet understood. The use of nucleotide binding as a regulatory feature in CPII would parallel other well-studied systems, while the bicarbonateinduced conformational changes in CPII relate to its dedicated role in regulating carbon concentration and carbon metabolism. Attempts were made to analyze the effects that binding partners might have on CPII, but as noted above, heterologous production of candidate CPII target effectors (the NDH-5-like protein, DUF2309, and the low-complexity transmembrane protein) were unsuccessful. A clearer picture of which other proteins directly interact with CPII, most likely in the fully-ADP-bound state 
promoted by bicarbonate binding, will be important in clarifying its cellular activities and its downstream signaling pathway. 


\section{METHODS}

Analysis of CPII Genes and their Genomic Context

A comparative genomics analysis of CPII was performed using the following online programs: SEED ${ }^{86}$, STRING $^{87}$, Clustal Omega ${ }^{88}, \mathrm{NCBI}$ protein domain search and NCBI protein BLAST tool. Sequences of canonical nrPII proteins used for comparisons to CPIls were extracted from phylogenetically distinct proteins with structures deposited in the Protein Data Bank. CPII proteins (NCBI protein ID from $T$. intermedia: ADG29527) were selected from operons that included both a DUF2309 protein (NCBI protein ID from T. intermedia: WP_013121928) and NADH:ubiquinone oxidoreductase like protein (NCBI protein ID from $T$. intermedia: WP_013121926) which together were near $\alpha$-carboxysome operons. The radial phylogenetic tree was generated using the online program Phylogeny.fr ${ }^{89}$ (http://phylogeny.lirmm.fr/phylo_cgi/index.cg) in August 2015. The radial phylogenetic tree of chosen nrPII and CPII sequences was generated with the phylogenetic analysis One Click method using default parameters.

\section{Cloning and Protein Expression of CPII}

A gBlock (purchased from Integrated DNA Technologies, Inc.) encoding the $T$. intermedia CPII amino acid sequence with a C-terminal 6-Histidine tag was assembled by the Gibson method ${ }^{90}$ into the plasmid pET28a between the $\mathrm{Ncol}$ and HindIII restriction enzyme sites. A sequence-verified clone was transformed into E. coli strain BL21(DE3) cells. Cells were grown overnight in $100 \mathrm{~mL}$ LB broth, $15 \mu \mathrm{M}$ kanamycin, with shaking at $200 \mathrm{rpm}$ at $37^{\circ} \mathrm{C}$. A $15 \mathrm{~mL}$ aliquot of the overnight culture was used to inoculate LB media in $1 \mathrm{~L}$ baffled flasks. These inoculates were grown with shaking at $37^{\circ} \mathrm{C}$ until the optical density, measured by absorbance $600 \mathrm{~nm}$, reached 0.8 . At this point, protein expression was induced by the addition of isopropyl- $\beta$-D-thiogalactoside (IPTG) to a final concentration of $1 \mathrm{mM}$, and shaken at $23^{\circ} \mathrm{C}$ for 24 hours. Afterwards, bacterial cell pellets were frozen and stored at $-20^{\circ} \mathrm{C}$. 
The 6His-SUMO-CPII construct was cloned so that a tagless version of CPII could be purified. CPIIencoding gBlocks were assembled into a pET-vector that had previously been engineered to contain an $\mathrm{N}$-terminal hexahistidine SUMO ${ }^{91}$-encoding gene. The N-terminal CPII methionine directly follows the two glycine residues recognized by ULP1 protease. Cleavage occurs C-terminal to the second glycine. 6His-SUMO-CPII was expressed similarly to 6His-CPII.

\section{Purification of CPII}

One liter of cells from $6 \mathrm{His}-\mathrm{CPII}$ were resuspended at $4^{\circ} \mathrm{C}$ in $50 \mathrm{~mL}$ the buffer $50 \mathrm{mM}$ Tris- $\mathrm{HCl} \mathrm{pH} \mathrm{7.6,} 300$ $\mathrm{mM} \mathrm{NaCl}$, one pill of Roche complete EDTA-free protease inhibitor cocktail, and lysed by sonication. Cell debris was pelleted by centrifugation at $16,500 \mathrm{rpm}$ in an SS-34 rotor for 30 minutes at $4^{\circ} \mathrm{C}$. The resulting supernatant was filtered through a $0.22 \mu \mathrm{m}$ Whatman membrane filter, washed with $30 \mathrm{~mL}$ wash-buffer (50 mM Tris- $\mathrm{HCl}$ pH 7.6, $300 \mathrm{mM} \mathrm{NaCl}, 100 \mathrm{mM}$ imidazole), and eluted in one step with elution buffer (50 mM Tris- $\mathrm{HCl} \mathrm{pH} \mathrm{7.6,} 300 \mathrm{mM} \mathrm{NaCl}, 500$ mM imidazole). Purified CPII was dialyzed into $10 \mathrm{mM}$ Tris- $\mathrm{HCl} \mathrm{pH} \mathrm{7.6,50} \mathrm{mM} \mathrm{NaCl}$. The first purification step of 6His-SUMO-CPII was performed as above. After elution of 6-His-SUMO-CPII, the fraction was diluted 6-fold with $50 \mathrm{mM}$ Tris- $\mathrm{HCl} \mathrm{pH} \mathrm{7.6,} 300$ $\mathrm{mM} \mathrm{NaCl}$ and incubated overnight at room temperature with approximately $1-3 \mathrm{mg}$ of $6 \mathrm{His}-\mathrm{ULP} 1$ (6Hisubiquitin like protease 1). The solution was then run through a nickel column equilibrated with $50 \mathrm{mM}$ Tris pH 7.6, $300 \mathrm{mM} \mathrm{NaCl}$. SDS-PAGE confirmed that the flow-through contained pure CPII. Samples were dialyzed into appropriate buffers, concentrated and stored at $4^{\circ} \mathrm{C}$ or $-20^{\circ} \mathrm{C}$ until further use.

\section{Protein Crystallization}

Hanging-drop crystallization screens were performed using the nanoliter liquid handling Mosquito from TTP LabTech at the High Throughput Macromolecular Crystallization Facility at UCLA. Crystallization conditions were screened using the JCSG, Structure Screen I and II and Wizard crystallization screens. 
For all trials, the concentrations of $6 \mathrm{His}-\mathrm{CPII}$ and CPII were between $25-30 \mathrm{mg} / \mathrm{mL}$ (2.0-2.5 mM CPII monomer) as determined by absorbance at $280 \mathrm{~nm}$ using the theoretical extinction coefficient 0.3480 $\mathrm{M}^{-1} \mathrm{~cm}^{-1}$. When other ligands were included in co-crystallization conditions, their concentrations were

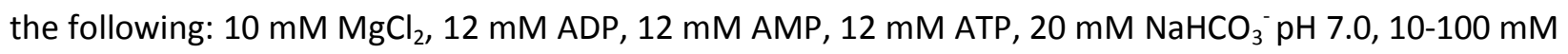
2-oxogluatarate $\mathrm{pH}$ 7.0. In order to control $\mathrm{pH}$ and prevent the possibility of $\mathrm{pH}$-dependent hydrolysis of nucleotides, solutions were buffered by $100 \mathrm{mM}$ Tris pH 7.2 and $50 \mathrm{mM} \mathrm{NaCl}$. When nucleotides were absent, buffers contained $10 \mathrm{mM}$ Tris $\mathrm{pH} 7.2$ and $50 \mathrm{mM} \mathrm{NaCl}$. Sodium bicarbonate and sodium $\alpha$ ketoglutarate solutions were prepared in $50 \mathrm{mM}$ Tris and titrated to a final $\mathrm{pH}$ of 7.2 with $\mathrm{NaOH}$. Sodium adenosine diphosphate stock solutions were prepared by resuspension of powdered sodium ADP (Sigma Aldrich). Final concentrations of ADP solutions were determined using a Nano-drop spectrophotometer at $260 \mathrm{~nm}$. The theoretical extinction coefficient we used for ADP is $15400 \mathrm{M}^{-1} \mathrm{~cm}^{-1}$. The final $\mathrm{pH}$ of ADP solutions were estimated using Millipore pH strips.

Optimizations of initial crystal hits were performed using an oil immersion sitting drop technique in 96 well plates. The technique employed was similar to batch crystallization, but with features allowing for variation in protein concentration and evaporation rates. Eight diluted solutions of CPII were prepared by mixing the original CPII protein stock with buffer to give final concentrations that were $94 \%, 88 \%$, $82 \%, 76 \%, 70 \%, 64 \%, 58 \%$, and $52 \%$ of the original value. These eight CPII diluted solutions were prepared along the $\mathrm{A}-\mathrm{H}$ axis in a single column of a 96-well plate. Using the TTP Laptech Mosquito Crystal machine, $400 \mathrm{nl}$ of the diluted range of CPII solutions were transferred from the reservoir column to each well of a Greiner microbatch plate. All 96 drops were then overlaid with $200 \mathrm{nl}$ of the original crystallization condition, which was obtained directly from the well of the original crystal screen condition. A gradient of paraffin-silicon oil mixtures, ranging from $100 \%$ paraffin to $9.1 \%$ paraffin in silicon oil, were prepared along the 12 columns of the 96-well microplate. A 12-channel pipette was 
used to overlay all 96 sitting-drops with $15 \mu \mathrm{L}$ of oil. The recessed wells of the microbatch plate enabled the oil to cover the drops without mixing between wells, so that the rate at which the drops lost water would vary along each row of the microplate.

\section{Phasing and Atomic Refinement}

Diffraction data were collected both in-house and at the Advanced Photon Source Argonne National Laboratory synchrotron. Our initial structure of apo-CPII was determined by molecular replacement using the SbtB structure from $T$. thermophilus H8, PDB ID 2CZ4, as a search model. The subsequent crystal structures were determined by molecular replacement using apo-CPII monomers as search models. Molecular replacement, model building and atomic refinement procedures were performed with $\mathrm{PHASER}^{92}, \mathrm{COOT}^{93}$, and $\mathrm{BUSTER}^{94}$ or Phenix ${ }^{95}$, respectively. Data quality and atomic refinement statistics are shown in Table I. A summary of deposited crystal structures and corresponding crystallization conditions is provided in Table S1.

\section{Site-Directed Spin Labeling, EPR Spectroscopy}

Purified protein containing an engineered cysteine mutation at site 109 was cloned using Gibson assembly with gBlock from ITD, and purified by nickel column as previously described. It was solvent exchanged with sample buffer $(25 \mathrm{mM}$ Tris- $\mathrm{HCl}$ pH 7.2, $200 \mathrm{mM} \mathrm{NaCl}$, and $10 \%$ glycerol) via a preequilibrated PD-10 desalting column from GE Lifesciences. The protein concentration was then determined by UV absorbance at $280 \mathrm{~nm}$ and an equimolar amount of spin label S-(1-oxy-2,2,5,5,tetramethylpyrroline-3-methyl)-methanethiosulfonate, more commonly referred to as MTSSL, was added and incubated at room temperature for 10 minutes to generate the nitroxide side chain designated $R 1^{84}$. After incubation, the sample was desalted with a freshly equilibrated PD-10 column and was then concentrated to approximately $800 \mu \mathrm{M}$ using a $10 \mathrm{KDa}$ concentrator and stored at $-20^{\circ} \mathrm{C}$ 
for subsequent preparations. Nucleotides were prepared at stock concentration of $100 \mathrm{mM}$ with a final $\mathrm{pH}$ of 7.5 as determined by Millipore $\mathrm{pH}$ strips.

All EPR Spectra were collected at room temperature using a Varian E-109 Model EPR spectrometer equipped with a 2-loop-1-gap Resonator at X-band microwave frequency. ${ }^{96}$ Continuous wave (CW) spectra were typically the average of 8 to 16 scans. Samples were typically 12 to $15 \mu \mathrm{L}$ in volume and consisted of $10 \mu \mathrm{M}$ spin labeled protein, sample buffer, and varying concentrations of nucleotide. To determine the fraction of nucleotide-bound protein in each sample, the spectra were fit using custom LabView software to weighted linear combinations of nucleotide free and nucleotide fully-bound lineshapes. (Supplemental Figure 1) A plot of the fraction bound versus nucleotide concentration was then fit to a two-state binding model to calculate the binding constant under each condition.

\section{Equilibrium Dialysis}

Purified CPII and ADP concentrations were measured immediately prior to experiment setup. Protein was placed in either 50ul or 100ul dialysis buttons (Hampton Research) in triplicate with or without $50 \mathrm{mM}$ sodium bicarbonate, respectively. Buttons were sealed with dialysis membranes (Spectrum Labs) possessing $6-8 \mathrm{kD} \mathrm{MW}$ cutoffs and incubated in $10 \mathrm{ml}$ volumes of nucleotide solution. The system was equilibrated at room temperature under conditions of gentle mixing by orbital rotation. After approximately sixteen hours, the solutions both inside and outside the dialysis button were assessed for nucleotide concentration using $A_{260}$ readings with a NanoDrop 2000 UV-Vis spectrophotometer (Thermo Scientific), being corrected for CPII $A_{260}$ contributions inside the button by subtracting values measured from a control dialysis equilibrium button prepared without nucleotide but containing identical protein concentrations. ADP concentration determined in the outer volume constitutes $[A D P]_{\text {free }}$ while the ADP concentration contained within the button represents $[A D P]_{\text {total }}$. Concentration of bound complex was 
calculated as $[C P I I-A D P]=[A D P]_{\text {total }}-[A D P]_{\text {free }} \cdot[C P I I]_{\text {total }}$ is user-defined the onset of the experiment and confirmed by $A_{280}$ measurements taken from the control dialysis equilibrium button. Free protein concentration was calculated as $[\mathrm{CPII}]_{\text {free }}=[\mathrm{CPII}]_{\text {total }}-[$ CPII-ADP $]$. The binding constant was calculated from a one-site binding model as $\mathrm{K}_{\mathrm{D}}=\left([\mathrm{CPII}]_{\text {free }} *[\mathrm{ADP}]_{\text {free }}\right) /[\mathrm{CPII-ADP}]$. Measurement uncertainty was determined by propagating fractional errors (estimated from triplicate measurement variations) throughout the calculations. 


\section{REFERENCES}

1. Andersson, I. Catalysis and regulation in RuBisCO. J. Exp. Bot. 59, 1555-1568 (2007).

2. Andersson, I. \& Backlund, A. Structure and function of RuBisCO. Plant Physiol. Biochem. PPB Société Fr. Physiol. Végétale 46, 275-291 (2008).

3. Martin, W. \& Schnarrenberger, C. The evolution of the Calvin cycle from prokaryotic to eukaryotic chromosomes: a case study of functional redundancy in ancient pathways through endosymbiosis. Curr. Genet. 32, 1-18 (1997).

4. Sims, P. A. 'Big-Picture' worksheets to help students learn and understand the Pentose Phosphate Pathway and the Calvin Cycle. J. Chem. Educ. 91, 541-545 (2014).

5. Spreitzer, R. J. \& Salvucci, M. E. Rubisco: structure, regulatory interactions, and possibilities for a better enzyme. Plant Biol. 53, (2002).

6. Bauwe, H., Hagemann, M., Kern, R. \& Timm, S. Photorespiration has a dual origin and manifold links to central metabolism. Curr. Opin. Plant Biol. 15, 269-275 (2012).

7. Kozaki, A. \& Takeba, G. Photorespiration protects C3 plants from photooxidation. Nature $\mathbf{3 8 4}$, 557560 (1996).

8. Wingler, A., Lea, P. J., Quick, W. P. \& Leegood, R. C. Photorespiration: metabolic pathways and their role in stress protection. Philos. Trans. R. Soc. Lond. B Biol. Sci. 355, 1517-1529 (2000).

9. Raven, J. A., Cockell, C. S. \& Rocha, C. L. D. L. The evolution of inorganic carbon concentrating mechanisms in photosynthesis. Philos. Trans. R. Soc. Lond. B Biol. Sci. 363, 2641-2650 (2008).

10. Smith, E. C. \& Griffiths, H. A pyrenoid-based carbon-concentrating mechanism is present in terrestrial bryophytes of the class Anthocerotae. Planta 200, 203-212 (1996).

11. Keeley, J. E. \& Rundel, P. W. Evolution of CAM and C4 carbon-concentrating mechanisms. Int. J. Plant Sci. 164, S55-S77 (2003). 
12. Espie, G. S. \& Kimber, M. S. Carboxysomes: cyanobacterial RuBisCO comes in small packages. Photosynth. Res. 109, 7-20 (2011).

13. Giordano, M., Beardall, J. \& Raven, J. A. $\mathrm{CO}_{2}$ concentrating mechanisms in algae: mechanisms, environmental modulation, and evolution. Annu. Rev. Plant Biol. 56, 99-131 (2005).

14. Badger, M. R. \& Price, G. D. $\mathrm{CO}_{2}$ concentrating mechanisms in cyanobacteria: molecular components, their diversity and evolution. J. Exp. Bot. 54, 609-622 (2003).

15. Ogawa, T. \& Kaplan, A. Inorganic carbon acquisition systems in cyanobacteria. Photosynth. Res. 77, 105-115 (2003).

16. Omata, T., Takahashi, Y., Yamaguchi, O. \& Nishimura, T. Structure, function and regulation of the cyanobacterial high-affinity bicarbonate transporter, BCT1. Funct. Plant Biol. 29, 151-159 (2002).

17. Price, G. D., Woodger, F. J., Badger, M. R., Howitt, S. M. \& Tucker, L. Identification of a SulP-type bicarbonate transporter in marine cyanobacteria. Proc. Natl. Acad. Sci. 101, 18228-18233 (2004).

18. Felce, J. \& Saier,Jr., M. H. Carbonic anhydrases fused to anion transporters of the SulP family: evidence for a novel type of bicarbonate transporter. J. Mol. Microbiol. Biotechnol. 8, 169-176 (2004).

19. Shibata, M. et al. Genes essential to sodium-dependent bicarbonate transport in cyanobacteria function and phylogenetic analysis. J. Biol. Chem. 277, 18658-18664 (2002).

20. López-Igual, R., Picossi, S., López-Garrido, J., Flores, E. \& Herrero, A. N and C control of ABC-type bicarbonate transporter $\mathrm{Cmp}$ and its LysR-type transcriptional regulator $\mathrm{CmpR}$ in a heterocyst-forming cyanobacterium, Anabaena sp. Environ. Microbiol. 14, 1035-1048 (2012).

21. Omata, T. et al. Identification of an ATP-binding cassette transporter involved in bicarbonate uptake in the cyanobacterium Synechococcus sp. strain PCC 7942. Proc. Natl. Acad. Sci. 96, 13571-13576 (1999).

22. Battchikova, N., Zhang, P., Rudd, S., Ogawa, T. \& Aro, E.-M. Identification of NdhL and Ssl1690 (NdhO) in NDH-1L and NDH-1M Complexes of Synechocystis sp. PCC 6803. J. Biol. Chem. 280, 2587-2595 (2005). 
23. Battchikova, N., Eisenhut, M. \& Aro, E.-M. Cyanobacterial NDH-1 complexes: Novel insights and remaining puzzles. Biochim. Biophys. Acta BBA - Bioenerg. 1807, 935-944 (2011).

24. Klughammer, B., Sültemeyer, D., Badger, M. R. \& Price, G. D. The involvement of NAD(P)H dehydrogenase subunits, $\mathrm{NdhD3}$ and NdhF3, in high-affinity $\mathrm{CO}_{2}$ uptake in Synechococcus sp. PCC7002 gives evidence for multiple NDH-1 complexes with specific roles in cyanobacteria. Mol. Microbiol. 32, 1305-1315 (1999).

25. Rae, B. D. et al. Cyanobacterial carboxysomes: microcompartments that facilitate $\mathrm{CO}_{2}$ fixation. J. Mol. Microbiol. Biotechnol. 23, 300-307 (2013).

26. Shively, J. M. \& English, R. S. The carboxysome, a prokaryotic organelle: a mini-review. Can. J. Bot. 69, 957-962 (1991).

27. Cannon, G. C. et al. Microcompartments in prokaryotes: carboxysomes and related polyhedra. Appl. Environ. Microbiol. 67, 5351-5361 (2001).

28. Yeates, T. O., Kerfeld, C. A., Heinhorst, S., Cannon, G. C. \& Shively, J. M. Protein-based organelles in bacteria: carboxysomes and related microcompartments. Nat. Rev. Microbiol. 6, 681-691 (2008).

29. Cai, F. et al. The pentameric vertex proteins are necessary for the icosahedral carboxysome shell to function as a $\mathrm{CO}_{2}$ leakage barrier. PLOS ONE 4, e7521 (2009).

30. Yeates, T. O., Thompson, M. C. \& Bobik, T. A. The protein shells of bacterial microcompartment organelles. Curr. Opin. Struct. Biol. 21, 223-231 (2011).

31. Wheatley, N. M., Gidaniyan, S. D., Liu, Y., Cascio, D. \& Yeates, T. O. Bacterial microcompartment shells of diverse functional types possess pentameric vertex proteins. Protein Sci. 22, 660-665 (2013).

32. Tanaka, S., Sawaya, M. R., Phillips, M. \& Yeates, T. O. Insights from multiple structures of the shell proteins from the $\beta$-carboxysome. Protein Sci. 18, 108-120 (2009).

33. Kerfeld, C. A. et al. Protein structures forming the shell of primitive bacterial organelles. Science 309, 936-938 (2005). 
34. Tanaka, S. et al. Atomic-level models of the bacterial carboxysome shell. Science 319, 1083-1086 (2008).

35. Tsai, Y. et al. Structural analysis of CsoS1A and the protein shell of the Halothiobacillus neapolitanus carboxysome. PLoS Biol. 5, e144 (2007).

36. Roberts, E. W., Cai, F., Kerfeld, C. A., Cannon, G. C. \& Heinhorst, S. Isolation and characterization of the Prochlorococcus carboxysome reveal the presence of the novel shell protein CsoS1D. J. Bacteriol. 194, 787-795 (2012).

37. Wheatley, N. M., Sundberg, C. D., Gidaniyan, S. D., Cascio, D. \& Yeates, T. O. Structure and identification of a pterin dehydratase-like protein as a RuBisCO assembly factor in the alphacarboxysome. J. Biol. Chem. jbc.M113.531236 (2014). doi:10.1074/jbc.M113.531236

38. Onizuka, T. et al. The rbcX gene product promotes the production and assembly of ribulose-1,5bisphosphate carboxylase/oxygenase of Synechococcus sp. PCC7002 in Escherichia coli. Plant Cell Physiol. 45, 1390-1395 (2004).

39. Baker et al. The correlation of the gene $\operatorname{csoS} 2$ of the carboxysome operon with two polypeptides of the carboxysome in Thiobacillus neapolitanus. Arch. Microbiol. 172, 233-239 (1999).

40. Peña, K. L., Castel, S. E., de Araujo, C., Espie, G. S. \& Kimber, M. S. Structural basis of the oxidative activation of the carboxysomal -carbonic anhydrase, CcmM. Proc. Natl. Acad. Sci. U. S. A. 107, 24552460 (2010).

41. Long, B. M., Badger, M. R., Whitney, S. M. \& Price, G. D. Analysis of carboxysomes from Synechococcus PCC7942 reveals multiple Rubisco complexes with carboxysomal proteins CcmM and CcaA. J. Biol. Chem. 282, 29323-29335 (2007).

42. Jorda, J., Lopez, D., Wheatley, N. M. \& Yeates, T. O. Using comparative genomics to uncover new kinds of protein-based metabolic organelles in bacteria. Protein Sci. 22, 179-95 (2012). 
43. Gaudana, S. B., Zarzycki, J., Moparthi, V. K. \& Kerfeld, C. A. Bioinformatic analysis of the distribution of inorganic carbon transporters and prospective targets for bioengineering to increase $\mathrm{Ci}$ uptake by cyanobacteria. Photosynth. Res. 126, 99-109 (2014).

44. Rae, B. D., Long, B. M., Badger, M. R. \& Price, G. D. Functions, compositions, and evolution of the two types of carboxysomes: polyhedral microcompartments that facilitate $\mathrm{CO} 2$ fixation in cyanobacteria and some proteobacteria. Microbiol. Mol. Biol. Rev. MMBR 77, 357-379 (2013).

45. Whitehead, L., Long, B. M., Price, G. D. \& Badger, M. R. Comparing the in vivo function of $\alpha-$ carboxysomes and $\beta$-carboxysomes in two model cyanobacteria. Plant Physiol. 165, 398-411 (2014).

46. Sutter, M. et al. Structural Characterization of a Newly Identified Component of $\alpha$-Carboxysomes: The AAA+ Domain Protein CsoCbbQ. Sci. Rep. 5, (2015).

47. Muro-Pastor, M. I., Reyes, J. C. \& Florencio, F. J. Cyanobacteria perceive nitrogen status by sensing intracellular 2-oxoglutarate levels. J. Biol. Chem. 276, 38320-38328 (2001).

48. Vázquez-Bermúdez, M. F., Herrero, A. \& Flores, E. 2-Oxoglutarate increases the binding affinity of the NtcA (nitrogen control) transcription factor for the Synechococcus glnA promoter. FEBS Lett. 512, 7174 (2002).

49. Doucette, C. D., Schwab, D. J., Wingreen, N. S. \& Rabinowitz, J. D. $\alpha$-ketoglutarate coordinates carbon and nitrogen utilization via enzyme I inhibition. Nat. Chem. Biol. 7, 894-901 (2011).

50. Hart, Y. et al. Robust control of nitrogen assimilation by a bifunctional enzyme in E. coli. Mol. Cell 41, 117-127 (2011).

51. Conroy, M. J. et al. The crystal structure of the Escherichia coli AmtB-GInK complex reveals how GInK regulates the ammonia channel. Proc. Natl. Acad. Sci. 104, 1213-1218 (2007).

52. Llácer, J. L. et al. Structural basis for the regulation of NtcA-dependent transcription by proteins PipX and PII. Proc. Natl. Acad. Sci. 107, 15397-15402 (2010). 
53. Llácer, J. L. et al. The crystal structure of the complex of PII and acetylglutamate kinase reveals how PII controls the storage of nitrogen as arginine. Proc. Natl. Acad. Sci. 104, 17644-17649 (2007).

54. Ninfa, A. J. \& Jiang, P. PII signal transduction proteins: sensors of $\alpha$-ketoglutarate that regulate nitrogen metabolism. Curr. Opin. Microbiol. 8, 168-173 (2005).

55. Forchhammer, K. \& Lüddecke, J. Sensory properties of the PIl signalling protein family. FEBS J. 283, 425-437 (2016).

56. Palanca, C. et al. The structure of a PII signaling protein from a halophilic archaeon reveals novel traits and high-salt adaptations. FEBS J. 281, 3299-3314 (2014).

57. Gundlach, J. et al. Identification, characterization, and structure analysis of the cyclic di-AMP-binding PII-like signal transduction protein DarA. J. Biol. Chem. 290, 3069-3080 (2015).

58. Adler, S. P., Purich, D. \& Stadtman, E. R. Cascade control of Escherichia coli glutamine synthetase. Properties of the PII regulatory protein and the uridylyltransferase-uridylyl-removing enzyme. J. Biol. Chem. 250, 6264-6272 (1975).

59. van Heeswijk, W. C. et al. An alternative PII protein in the regulation of glutamine synthetase in Escherichia coli. Mol. Microbiol. 21, 133-146 (1996).

60. Coutts, G. Membrane sequestration of the signal transduction protein GInK by the ammonium transporter AmtB. EMBO J. 21, 536-545 (2002).

61. Dodsworth, J. A. \& Leigh, J. A. Nifl inhibits nitrogenase by competing with Fe protein for binding to the MoFe protein. Biochem. Biophys. Res. Commun. 364, 378-382 (2007).

62. Drepper, T. Role of GlnB and GInK in ammonium control of both nitrogenase systems in the phototrophic bacterium Rhodobacter capsulatus. Microbiology 149, 2203-2212 (2003).

63. Hisbergues, M., Jeanjean, R., Joset, F., Tandeau de Marsac, N. \& Bédu, S. Protein PII regulates both inorganic carbon and nitrate uptake and is modified by a redox signal in Synechocystis PCC 6803. FEBS Lett. 463, 216-220 (1999). 
64. Feria Bourrellier, A. B. et al. Chloroplast acetyl-CoA carboxylase activity is 2-oxoglutarate-regulated by interaction of PII with the biotin carboxyl carrier subunit. Proc. Natl. Acad. Sci. U. S. A. 107, 502-507 (2010).

65. Gerhardt, E. C. M. et al. The bacterial signal transduction protein GInB regulates the committed step in fatty acid biosynthesis by acting as a dissociable regulatory subunit of acetyl-CoA carboxylase. Mol. Microbiol. 95, 1025-1035 (2015).

66. Lee, H. M., Flores, E., Forchhammer, K., Herrero, A. \& Tandeau De Marsac, N. Phosphorylation of the signal transducer PII protein and an additional effector are required for the PII-mediated regulation of nitrate and nitrite uptake in the Cyanobacterium Synechococcus sp. PCC 7942. Eur. J. Biochem. FEBS 267, 591-600 (2000).

67. Palinska, K. A. et al. The signal transducer PII and bicarbonate acquisition in Prochlorococcus marinus PCC 9511, a marine cyanobacterium naturally deficient in nitrate and nitrite assimilation. Microbiology 148, 2405-2412 (2002).

68. Du, J., Förster, B., Rourke, L., Howitt, S. M. \& Price, G. D. Characterisation of cyanobacterial bicarbonate transporters in E. coli shows that SbtA homologs are functional in this heterologous expression system. PLoS ONE 9, e115905 (2014).

69. Erhardt, H. et al. Disruption of individual nuo-genes leads to the formation of partially assembled NADH:ubiquinone oxidoreductase (Complex I) in Escherichia coli. Biochim. Biophys. Acta BBA Bioenerg. 1817, 863-871 (2012).

70. Hrdy, I. et al. Trichomonas hydrogenosomes contain the NADH dehydrogenase module of mitochondrial complex I. Nature 432, 618-622 (2004).

71. Xu, Y. et al. GInK, a PII-homologue: structure reveals ATP binding site and indicates how the T-loops may be involved in molecular recognition. J. Mol. Biol. 282, 149-165 (1998). 
72. Jiang, P. et al. Structure/function analysis of the PII signal transduction protein of Escherichia coli: genetic separation of interactions with protein receptors. J. Bacteriol. 179, 4342-4353 (1997).

73. Bonatto, A. C., Souza, E. M., Pedrosa, F. O., Yates, M. G. \& Benelli, E. M. Effect of T- and C-loop mutations on the Herbaspirillum seropedicae GInB protein in nitrogen signalling. Res. Microbiol. 156, 634-640 (2005).

74. Shetty, N. D., Reddy, M. C. M., Palaninathan, S. K., Owen, J. L. \& Sacchettini, J. C. Crystal structures of the apo and ATP bound Mycobacterium tuberculosis nitrogen regulatory PII protein. Protein Sci. 19, 1513-1524 (2010).

75. Xu, Y. et al. The structures of the PII proteins from the cyanobacteria Synechococcus sp. PCC 7942 and Synechocystis sp. PCC 6803. Acta Crystallogr. D Biol. Crystallogr. 59, 2183-2190 (2003).

76. Fokina, O., Chellamuthu, V.-R., Zeth, K. \& Forchhammer, K. A novel signal transduction protein P(II) variant from Synechococcus elongatus PCC 7942 indicates a two-step process for NAGK-P(II) complex formation. J. Mol. Biol. 399, 410-421 (2010).

77. Fokina, O., Chellamuthu, V.-R., Forchhammer, K. \& Zeth, K. Mechanism of 2-oxoglutarate signaling by the Synechococcus elongatus PII signal transduction protein. Proc. Natl. Acad. Sci. U. S. A. 107, 1976019765 (2010).

78. Yildiz, Ö., Kalthoff, C., Raunser, S. \& Kühlbrandt, W. Structure of GlnK1 with bound effectors indicates regulatory mechanism for ammonia uptake. EMBO J. 26, 589-599 (2007).

79. Rajendran, C. et al. Crystal structure of the GInZ-DraG complex reveals a different form of PII-target interaction. Proc. Natl. Acad. Sci. 108, 18972-18976 (2011).

80. Xu, Y., Carr, P. D., Huber, T., Vasudevan, S. G. \& Ollis, D. L. The structure of the PII-ATP complex. Eur. J. Biochem. 268, 2028-2037 (2001).

81. Heeswijk, W. C. van, Westerhoff, H. V. \& Boogerd, F. C. Nitrogen assimilation in Escherichia coli: putting molecular data into a systems perspective. Microbiol. Mol. Biol. Rev. 77, 628-695 (2013). 
82. Ninfa, A. J. \& Atkinson, M. R. Pll signal transduction proteins. Trends Microbiol. 8, 172-179 (2000).

83. Dror, R. O. et al. Signal transduction. Structural basis for nucleotide exchange in heterotrimeric G proteins. Science 348, 1361-1365 (2015).

84. Hubbell, W. L., Cafiso, D. S. \& Altenbach, C. Identifying conformational changes with site-directed spin labeling. Nat. Struct. Mol. Biol. 7, 735-739 (2000).

85. Albe, K. R., Butler, M. H. \& Wright, B. E. Cellular concentrations of enzymes and their substrates. J. Theor. Biol. 143, 163-195 (1990).

86. Overbeek, R. et al. The subsystems approach to genome annotation and its use in the project to annotate 1000 genomes. Nucleic Acids Res. 33, 5691-5702 (2005).

87. Jensen, L. J. et al. STRING 8--a global view on proteins and their functional interactions in 630 organisms. Nucleic Acids Res. 37, D412-416 (2009).

88. Sievers, F. et al. Fast, scalable generation of high-quality protein multiple sequence alignments using Clustal Omega. Mol. Syst. Biol. 7, 539-539 (2014).

89. Dereeper, A. et al. Phylogeny.fr: robust phylogenetic analysis for the non-specialist. Nucleic Acids Res. 36, W465-W469 (2008).

90. Gibson, D. G. et al. Enzymatic assembly of DNA molecules up to several hundred kilobases. Nat. Methods 6, 343-345 (2009).

91. Malakhov, M. P. et al. SUMO fusions and SUMO-specific protease for efficient expression and purification of proteins. J. Struct. Funct. Genomics 5, 75-86 (2004).

92. McCoy, A. J. et al. Phaser crystallographic software. J. Appl. Crystallogr. 40, 658-674 (2007).

93. Emsley, P. \& Cowtan, K. Coot: model-building tools for molecular graphics. Acta Crystallogr. D Biol. Crystallogr. 60, 2126-2132 (2004).

94. Bricogne, G. et al. BUSTER version X.Y.Z. Cambridge, United Kingdom: Global Phasing Ltd. (2011). 
95. Adams, P. D. et al. PHENIX: a comprehensive Python-based system for macromolecular structure solution. Acta Crystallogr. D Biol. Crystallogr. 66, 213-221 (2010).

96. Hubbell, W. L., Froncisz, W. \& Hyde, J. S. Continuous and stopped flow EPR spectrometer based on a loop gap resonator. Rev. Sci. Instrum. 58, 1879-1886 (1987). 


\section{FIGURE LEGENDS}

Figure 1. Conserved genomic associations for CPII, a novel type of PII regulatory protein in bacteria.

A. A schematic diagram of the $\mathrm{CO}_{2}$ concentrating mechanisms in bacteria including the carboxysome. For comparison to panel $\mathrm{A}$, the $\alpha$-carboxysome operons encode the RuBisCO and carbonic anhydrase (CA) enzymes along with multiple paralogs of the carboxysome shell proteins (shown as small blue genes in panel A). B. The CPII gene (denoted by an asterisk) occurs as part of a conserved NDH-PII gene cluster near the following: $\alpha$-carboxysome operons, pyruvate:ferredoxin oxidoreductase (PFOR) genes, and putative bicarbonate transporter systems SbtAB. PFOR genes are represented in shades of blue. The putative gene for SbtA is colored dark blue and appears adjacent SbtB, colored yellow. Representative gene clusters were selected for illustration among a large number of diverse cases. Operon diagrams were drawn from the following organisms, from top to bottom: Thiomonas intermedia, Acidithiobacillus caldus, Nitrosomonas eutropha, Halothiobacillus neapolitanus, Nitrococcus mobilis, Hydrogenivirga, Sulfurihydrogenibium yellowstonense, Sulfurihydrogenibium azorense and Roseiflexus castenholzii. "N" marks the position of the NDH-5 homolog, "L", the low complexity protein, "D" the DUF2309 protein and, asterisk $(*)$, the location of CPII. In the last case (Roseiflexus castenholzii), the NHD-5 and DUF2309 are encoded near SbtA and SbtB homologs without a low complexity "L" or CPII protein. C. A phylogenetic tree of representative protein sequences of PII and PII-like sequences. CPII forms a cluster separate from nrPII proteins. A star $(\star)$ marks the protein described in this work from Thiomonas intermedia.

Figure 2. Crystal structure of Apo-CPII from Thiomonas intermedia. A. A schematic diagram of the CPII monomer in an illustrative extended configuration to emphasize the secondary structure and key tertiary interactions in the folded state. Key secondary structure motifs are labeled. B. The apo-CPII 
trimer (PDB ID 5D4L) is shown as cartoons from the top and side views, with select motifs and residues labeled. Phe-57 is indicated by an asterisk (*).

Figure 3. Comparison of secondary and tertiary structures of CPII and nrPII A. Protein sequence alignments of select CPII and nrPII proteins. For the Thiomonas intermedia CPII sequence, secondary structural motifs are shown as ' $b$ ' for beta-sheet, ' $h$ ' for alpha-helix and '-' for coil. Conserved motifs of the CPII T-loop, B-loop and C-loop are colored green, blue and purple, respectively. From top to bottom, the sequences correspond to CPIIs from Thiomonas intermedia K12, Halothiobacillus neapolitanus C2, Nitrosomonas eutropha C91, Nitrobacter sp. Nb-311a, nrPIls from Bacillus subtilis, Synechocystis sp PCC 6803, Archaeologlobus fulgidus. B-E. Apo-CPII (PDB 5D4L) is aligned to nrPII of Synechococcus sp. PCC 6803 (PDB 1QY7). B. CPII's B-loop with conserved residues GSH shown in sticks is significantly shorter than the nrPII B-loop. C. The C-loop in nrPII is shown in dark blue. No analogous C-loop is present in apoCPII .D. Asn-41 in CPII contributes to the $\beta$-bulge, which is generally not present in nrPIl proteins. E. Two consecutive $90^{\circ}$ rotations of CPII in superposition with nrPII.

Figure 4. Nucleotide binding is associated with T-loop and C-loop conformational changes in the CPII trimer. Crystal structures are arranged in rows, viewed from the top, and from the side at three $120^{\circ}$ rotations, showing all three nucleotide binding clefts, which are sometimes different within the same trimer. In the top row, apo-CPII exemplifies the respective orientations shown in each column. T-loops are colored purple with Phe-57 residues shown in stick form. C-loops are colored yellow, with Lys-105 and Arg-102 shown in stick form. Bicarbonate and acetate are shown as spheres. The following three rows show the diversity of subunit and loop conformations in CPII in multiple distinct crystal structures with ligands present in some or all of the available binding sites. Panels A1-3 show the three ADP- and bicarbonate-bound clefts of the CPII trimer (PDB 5D4P). Note that all three T-loops are elevated above 
the plane of the CPII trimer, and all three C-loops are stabilized in an $\alpha$-helical conformation. B1-3 show the nucleotide binding clefts in PDB structure 5D4N. The clefts shown in B1, B2 and B3 are occupied by ADP and acetate, AMP and acetate, and no ligands, respectively. C1-3 show the three nucleotide binding clefts in PDB structure 5D40, bound to ADP and bicarbonate, AMP and bicarbonate, and no ligand, respectively. The nucleotides are shown in stick representation.

Figure 5. Conformational changes in the T-loop and C-loop upon ligand binding. T-loops are colored yellow with Phe-57 shown in stick form. C-loop residues are colored dark blue with Lys-105 shown in stick form. A. Stereo view of apo-CPII (PDB 5D4L). B. Stereo view of ADP- and acetate- bound CPII (PDB 5D4N).

Figure 6. Bicarbonate and acetate interact with Arg-102, a conserved residue in the C-terminal helix motif in CPII. A. The ADP-bound cleft (PDB 5DRK) (without bicarbonate) shows Arg-102 in the C-terminal helix motif (yellow) extending towards the solvent. B. The ADP and bicarbonate-bound cleft in PDB structure 5D40 shows Arg-102 positioned for interaction with bicarbonate. C. Overlay of panels A and B. D. The AMP-bound cleft in PDB structure 5DS7 (without bicarbonate) shows Arg-102 pointed towards solvent. E. In the AMP and bicarbonate-bound cleft (PDB 5D4O), Arg-102 is elevated towards the interior of the cleft, coordinating bicarbonate. F. Overlay of panes D and E. For all panels, the C-terminal segment (residues G101-R107) is colored yellow. R102, K105 and nucleotides are shown in sticks. Bicarbonate is shown in spheres.

\section{Figure 7. The affinity of CPII-R1 for ADP and AMP is influenced by bicarbonate.}

A. Continuous Wave (CW) EPR spectra of spin labeled CPII (C108R1) titrated with ADP and AMP. Incubation of $10 \mu \mathrm{M}$ CPII C108R1 with increasing concentrations of ADP or AMP drives an unstructured- 
to-structured transition at the protein's C-terminus; consistent with the formation of a C-terminal $\alpha$ helix seen in the nucleotide bound crystal structure. All spectra have been normalized and the structural transition can be seen as a broadening of the line-shape profile as nucleotide concentration increases. Line-shape broadening reflects restricted local motion of the spin labeled residue C109-R1 as the nucleotide binds and the C-terminus becomes structured. B. Titrating $10 \mu \mathrm{M}$ CPII-R1 with ADP under various concentrations of sodium bicarbonate reveals a bicarbonate induced increase in binding affinity of CPII for ADP. A representative set of titrations are plotted to illustrate this shift under $10 \mu \mathrm{M}$ (green), $5 \mathrm{mM}$ (yellow), $10 \mathrm{mM}$ (blue), and $50 \mathrm{mM}$ (orange) sodium bicarbonate conditions. Experiments were performed at room temperature (298 K). C. $10 \mu \mathrm{M}$ CPII-R1 was titrated with sodium bicarbonate in the presence of $2 \mathrm{mM}$ ADP (the approximate $K_{D}$ of $C P I$ and $A D P$ ). Increasing the concentration of bicarbonate stabilizes the ADP bound conformation of CPII and increases the binding affinity of CPII for ADP.

Figure 8. Electron density maps reveal features that can be unambiguously modeled by ADP, AMP and bicarbonate. Difference electron density maps are shown calculated as 'omit maps', with atoms belonging to the ligands removed from the phase calculation. Different binding sites (in crystal structure PDB ID 5D40) showing occupancy of bicarbonate and either ADP (top) or AMP (bottom) are illustrated in stereo view at contour levels of 5 (left) and 2.5 (right) standard deviations above the mean density value.

\section{Acknowledgements}

This work was supported by NIH grant AI081146 to T.O.Y. and by a UCLA Whitcome Fellowship to N.M.W. The authors thank Dean Price, David Savage and Avi Flamholz for their helpful suggestions. The 
UCLA X-ray diffraction facilities are supported by the BER program of the Department of Energy Office of Science under award DE-FC02-02ER63421. This work is based upon research conducted at the Northeastern Collaborative Access Team beamlines, which are funded by the National Institute of General Medical Sciences from the National Institutes of Health (P41 GM103403). The Pilatus 6M detector on 24-ID-C beam line is funded by a NIH-ORIP HEI grant (S10 RR029205). This research used resources of the Advanced Photon Source, a U.S. Department of Energy (DOE) Office of Science User Facility operated for the DOE Office of Science by Argonne National Laboratory under Contract No. DEAC02-06CH11357. We appreciate the expert help from the APS 24-ID-C beamline staff, including Kanagalaghatta Rajashankar, Malcolm Capel, Igor Kourinov, Jonathan Schuermann, and Narayanasami Sukumar. 

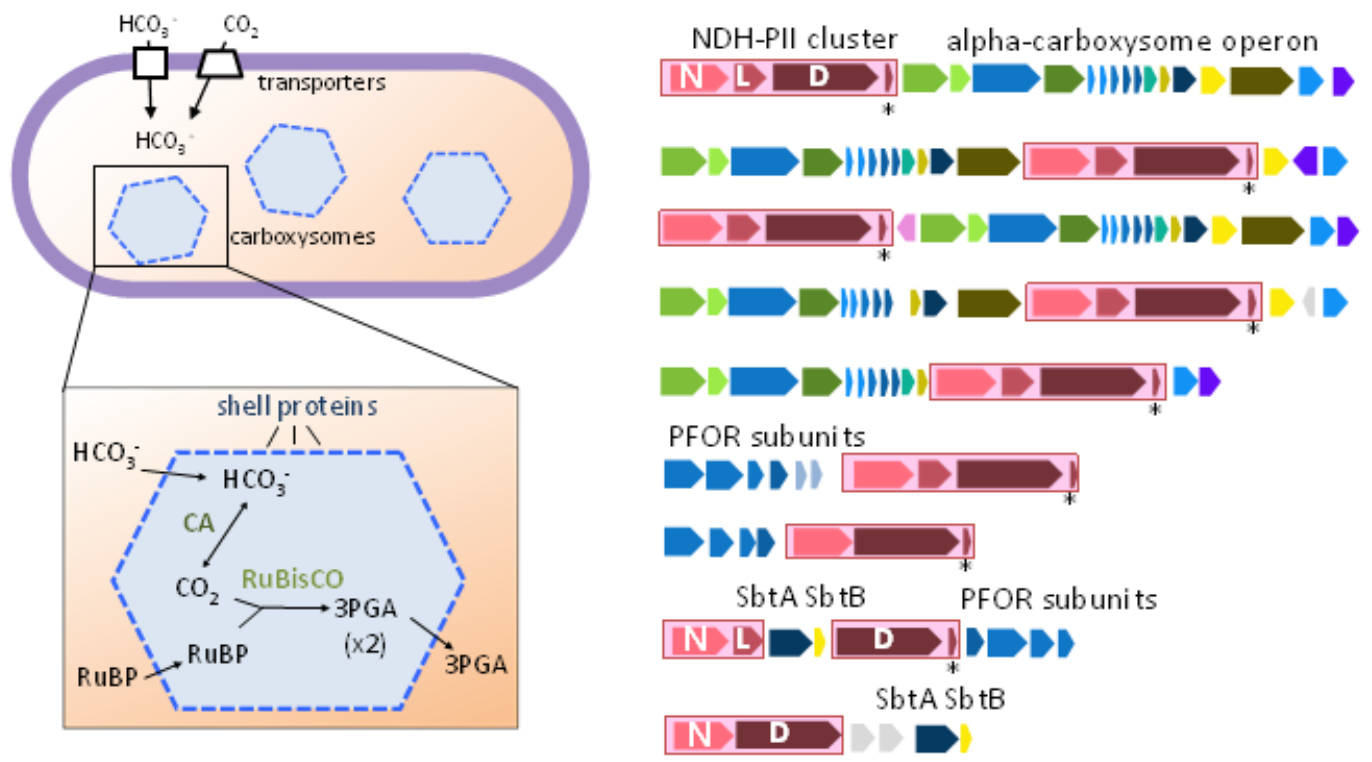

C

Cyanobacteria_Synechocystis_sp._PCC6803

Cyanobacteria_Anabaena_sp._PCC7120

[Cyanobacteria_Synechococcus_elongatus_PCC7942

-Eukaryote_Arabidopsis_lyrata

Proteobacteria_Escherichia_coli_GlnB

Firmicute_Mycobacterium_tuberculosis

nrPII

Proteobacteria_Escherichia_coli_GlnK

Archaea_Archaeoglobus_fulgidus_Glnk3

Archaea_Archaeoglobus_fulgidus_GlnK2

- Archaea_Haloferax_mediterranei

_Fimicute_Bacillus_subtilis

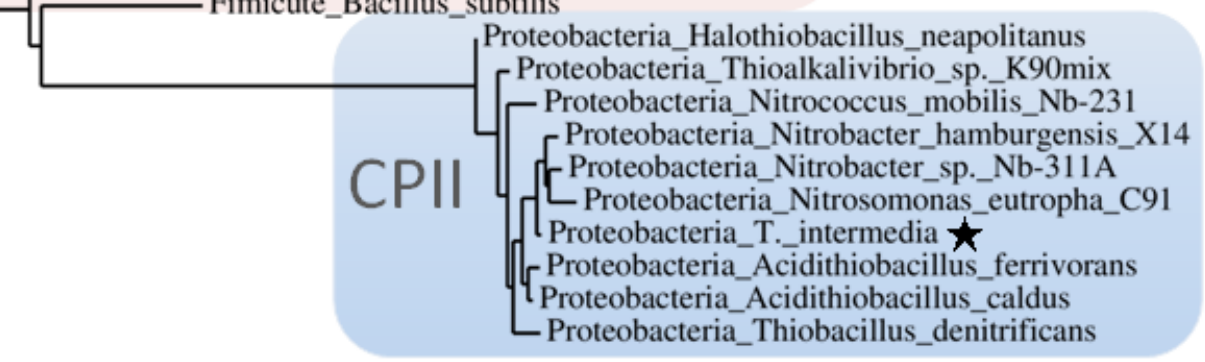

FIGURE 1 

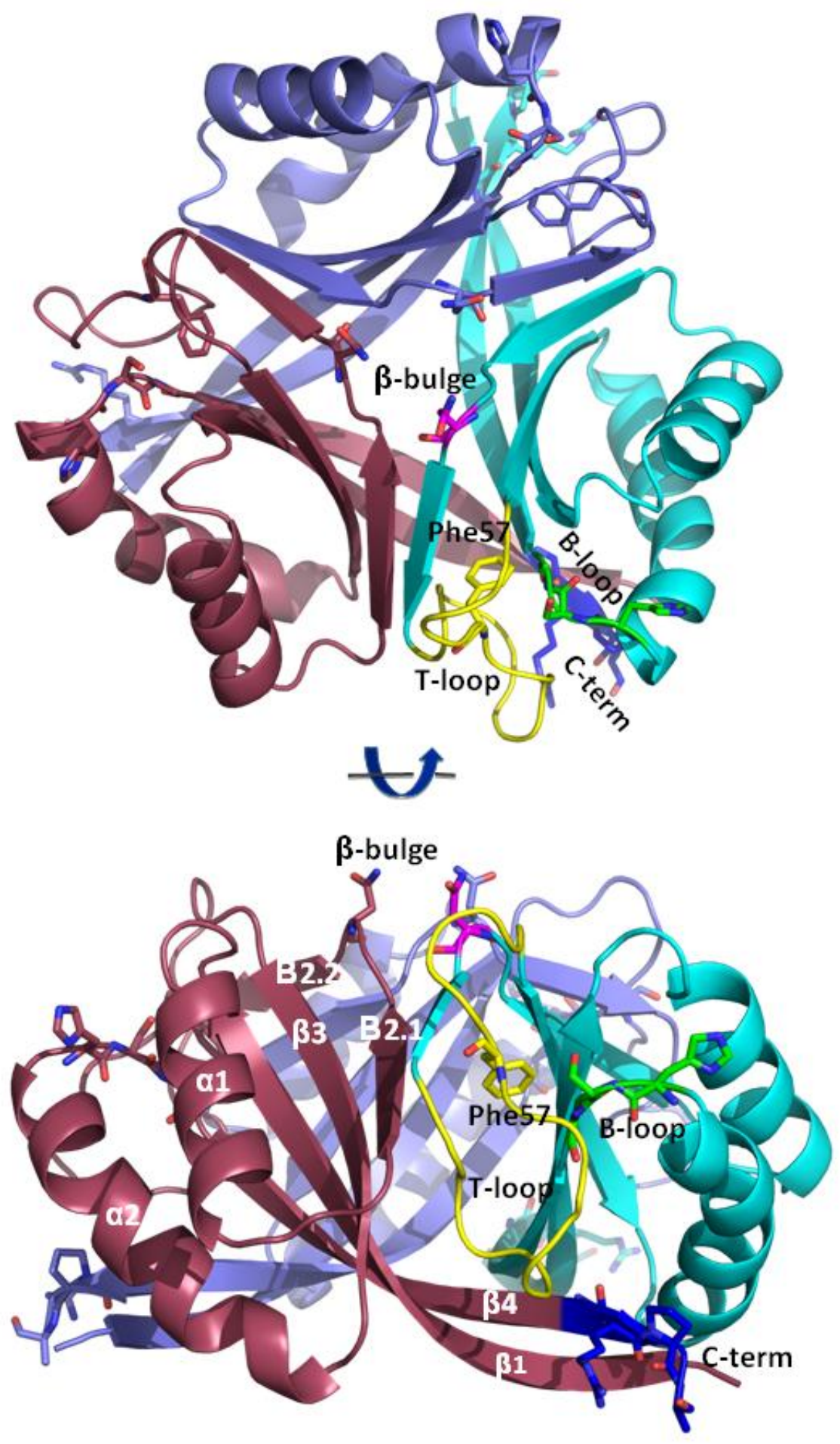

FIGURE 2 
1

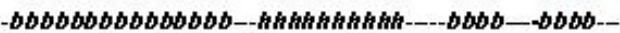

10

20

$30 \quad 40$

50

1

CPII MKNLNLTQLKKLEIILEGEHQSF ATDLLDR AG WKG YTIVGNLSGKGAQ--.-GFHEGHLM

CPIII MSTF KLSPLKKLEIILAGE HREF AT SLLDR ADVKG YTIVGNLSGKGSQ----GFYGGHLM

CPIII MNE IKLLPLKKLEIILQGEHKEFATDLLDRAGVKGYTIIGNLSGKGAH----GMYEGHLM

NYPIII-----MFKVEIVTRP ANFEKLKQELGKIGVTSLTFS-N VHGCGLQKAHTELYRGVKI

NrPIII------MKKVEAIR PFKLDE WI AL WN AGIVGMTVS-EVRGFGRQKGQTERYRGSEY

NrPIII--.---MKMWAYRP EKLECVKKALEERGFVGMTVT -E WGRGEQKGIRLQFRGREV $:: \ldots^{*} \ldots{ }^{*}::^{* *}:{ }^{*}$

...T-loop

B-loop

C-loop

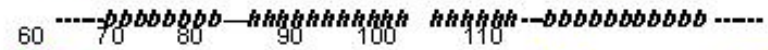

CPIII FIIED D ALIMII AAYPEEL VGPLLEGF -.-QPFFEAHSGWF VHDIQVGR-PIKFRN-.-

CPIII FNEDE ENM MIIT AYPEEL VSPLLEGF ---APFF SKHSG WV VSDIQVSR -P WF KTDAKK

CPIII FNEDD ALIMII VA VSPNL WD ALLEGF ---QPFFEAHSG MFVSDIQVGR--PVKFMA--

CPIII FNEDD MIMIIIAAYPQNLFD PLLEGF -.-_QPFFEAHSG MF VSDIQVGR --LVKFVA.--

NYPIIIESN WERLKIEIWSKYPVDQVTET AKRVLKTGSPGDGKIF WYEISNTPE ALINIRT---

NrPIIITEFLQKLKIEI WDEGQVDM WD KLVSAARTGEIGDGKIFISPVD SV----VRIRTGEK

NrPIIEVDLLQKTKYE WWSDD AVDEVVE AVVSARTGKFGDGRIF MPVEKS----WIRTGDE

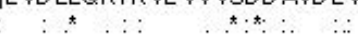

B

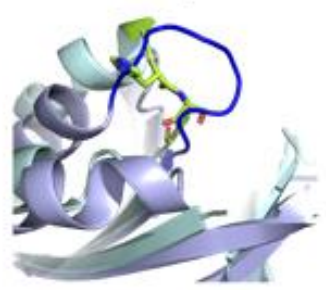

E

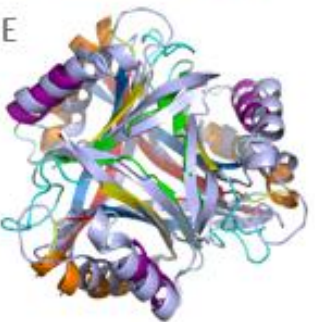

C C-loop
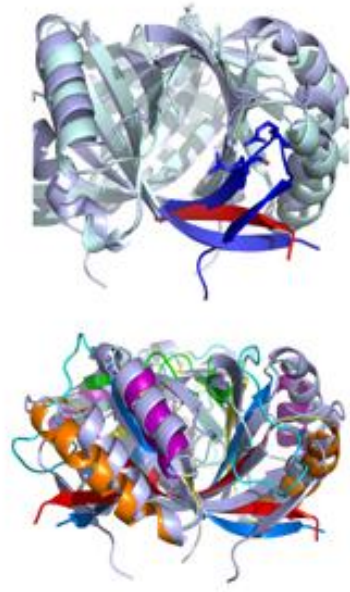

D $\beta$-bulge

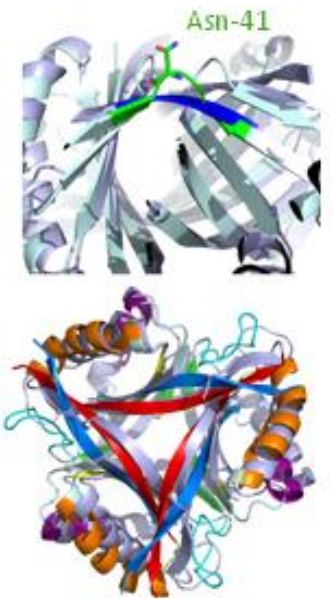

FIGURE 3 

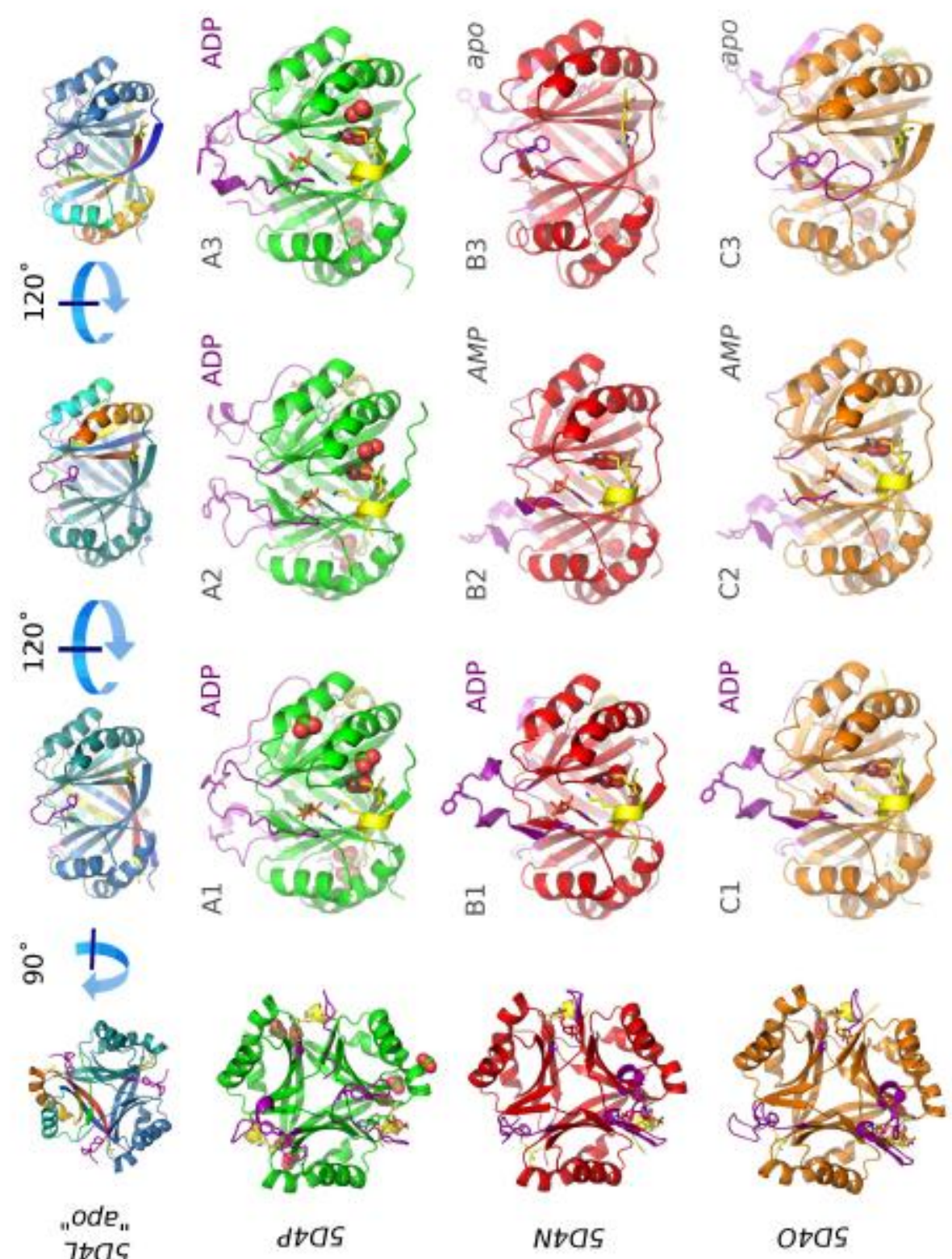

Figure 4 

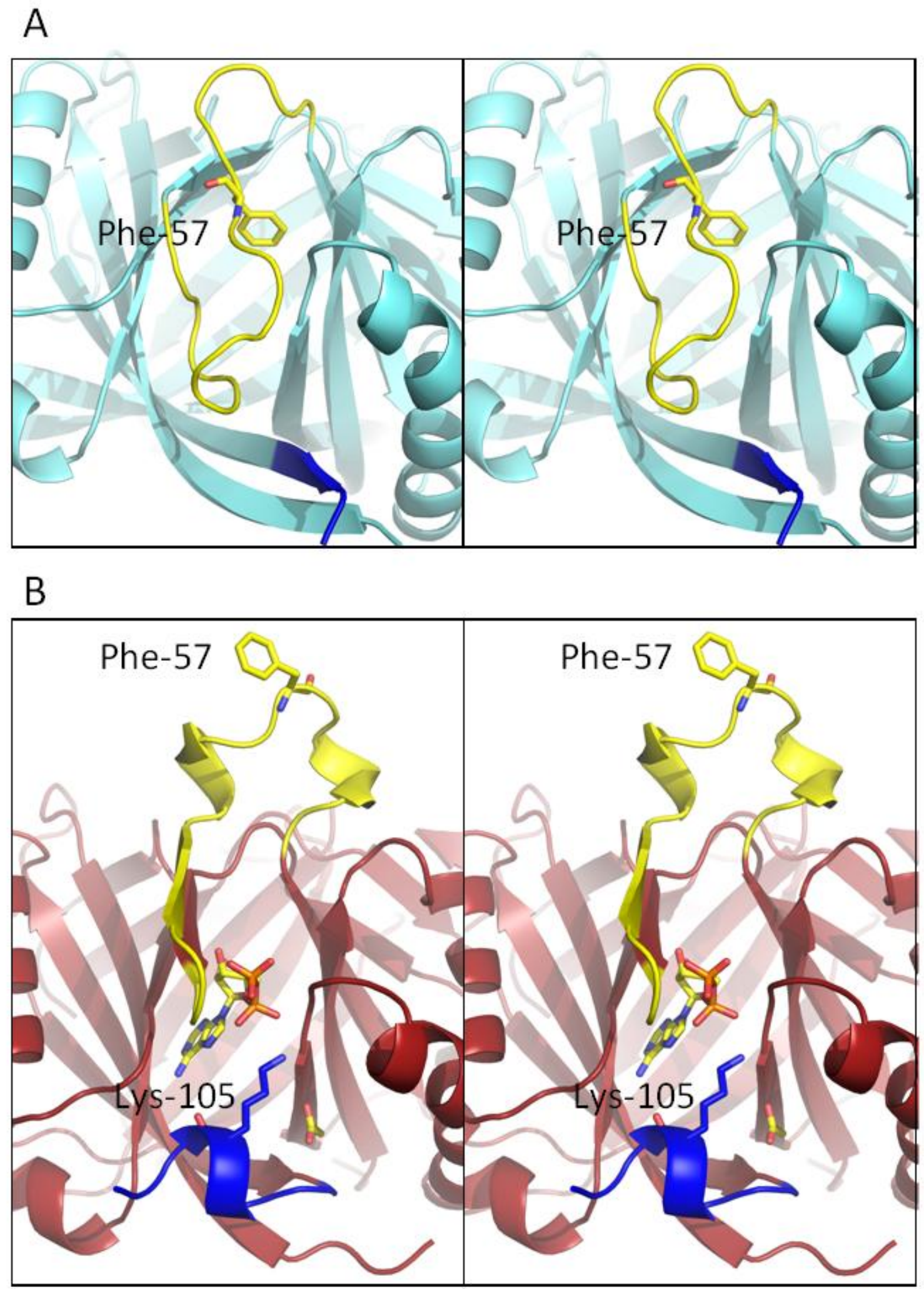

Figure 5 


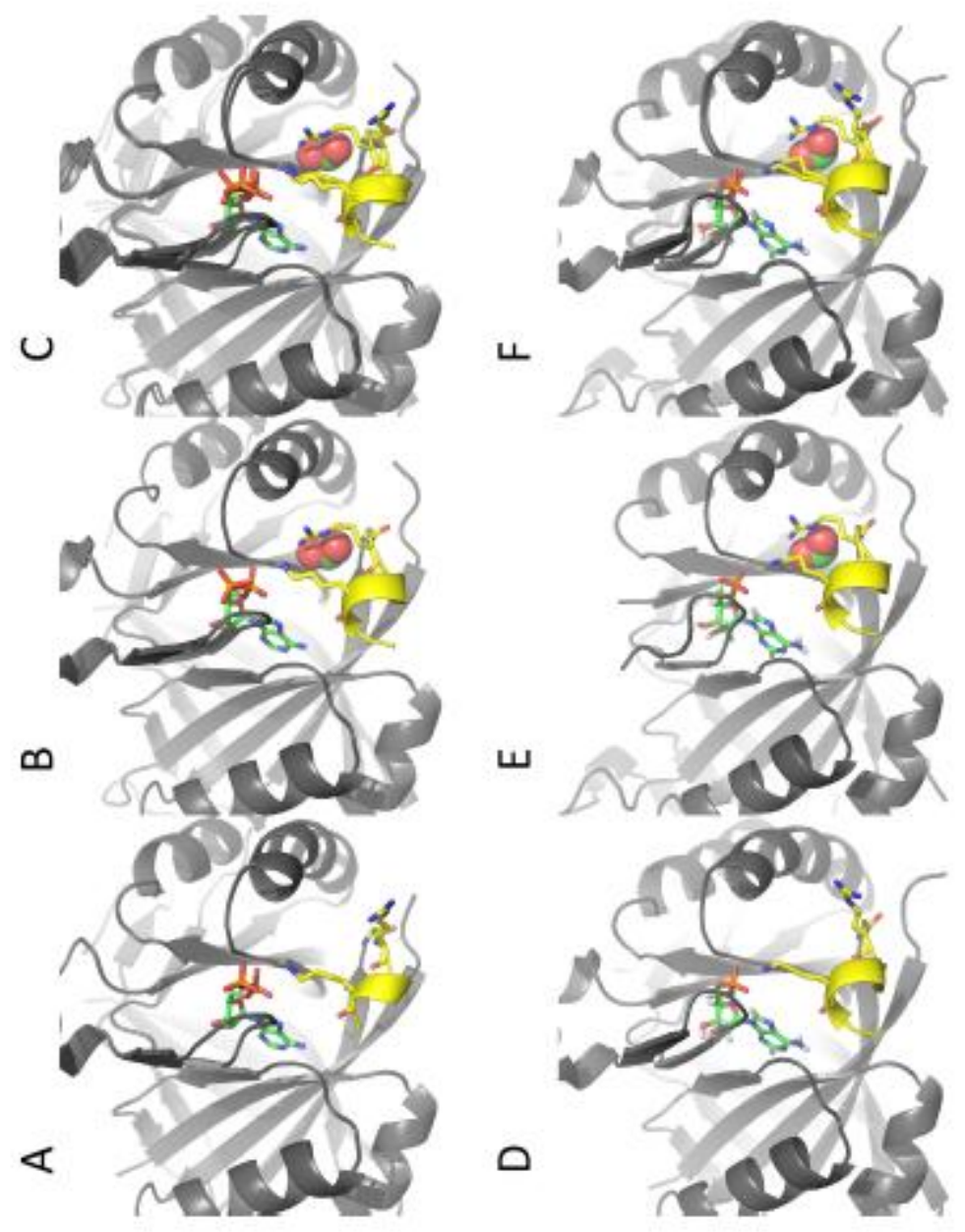

Figure 6 
CIPII-R1 EPR spectra with increasing nucleotide concentrations
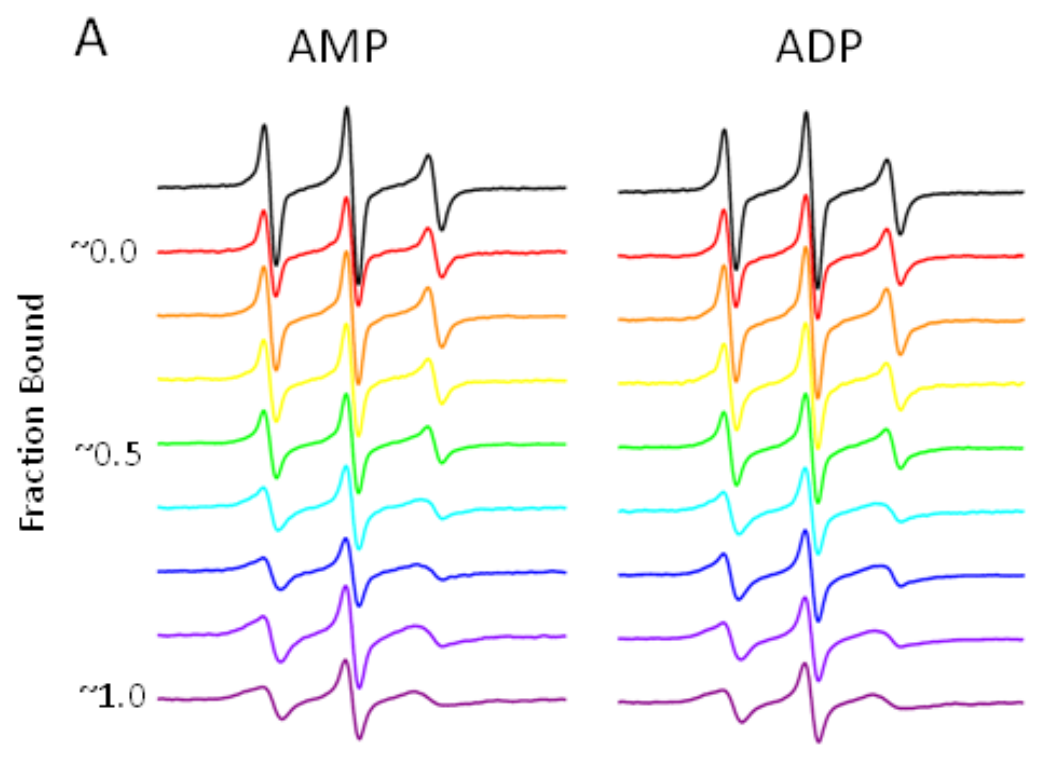

[Nucleotide]

CPII-R1 nucleotide affinity is dependent on bicarbonate $\left(\mathrm{HCO}_{3}{ }^{-}\right)$

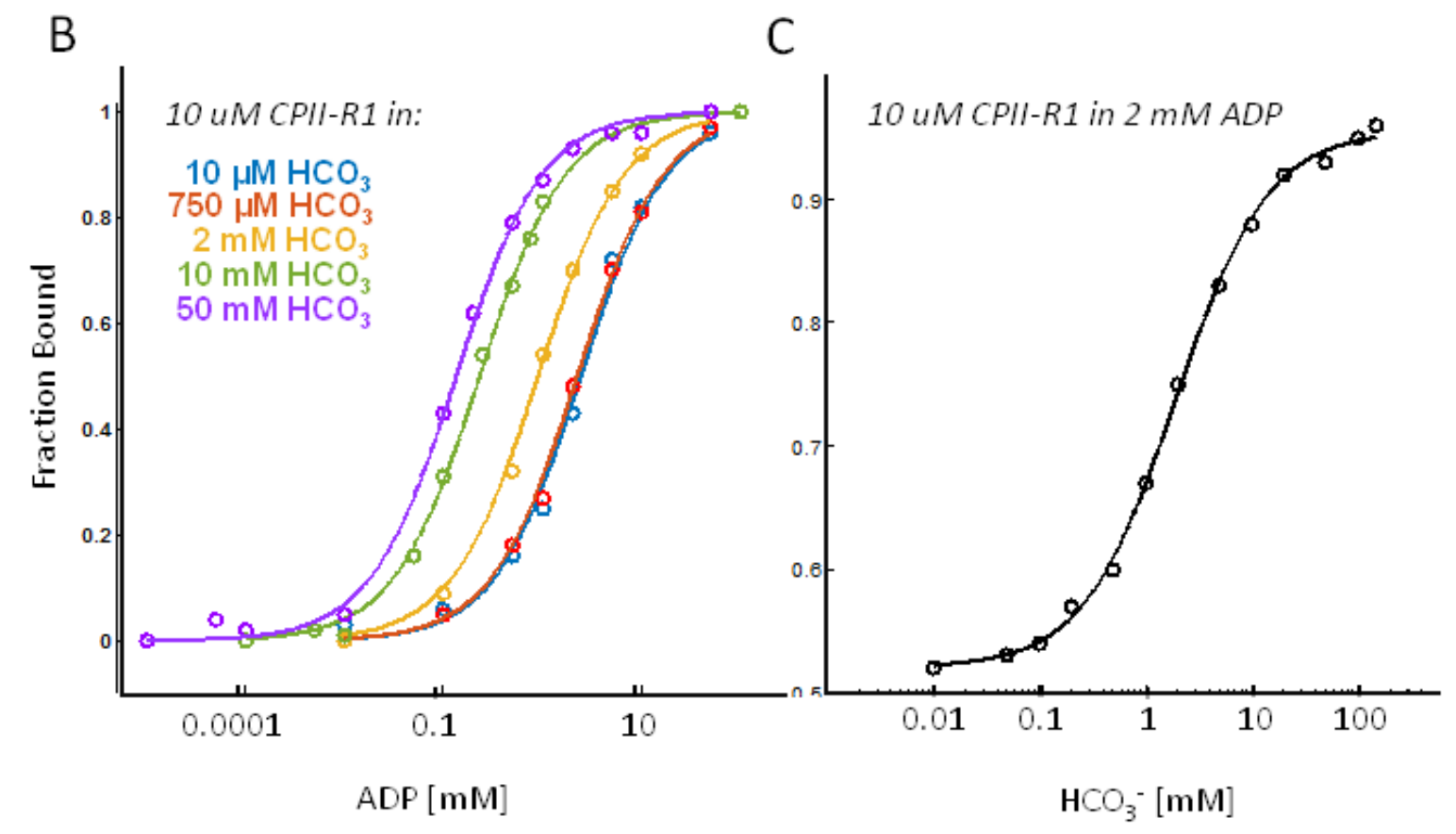

C

FIGURE 7 

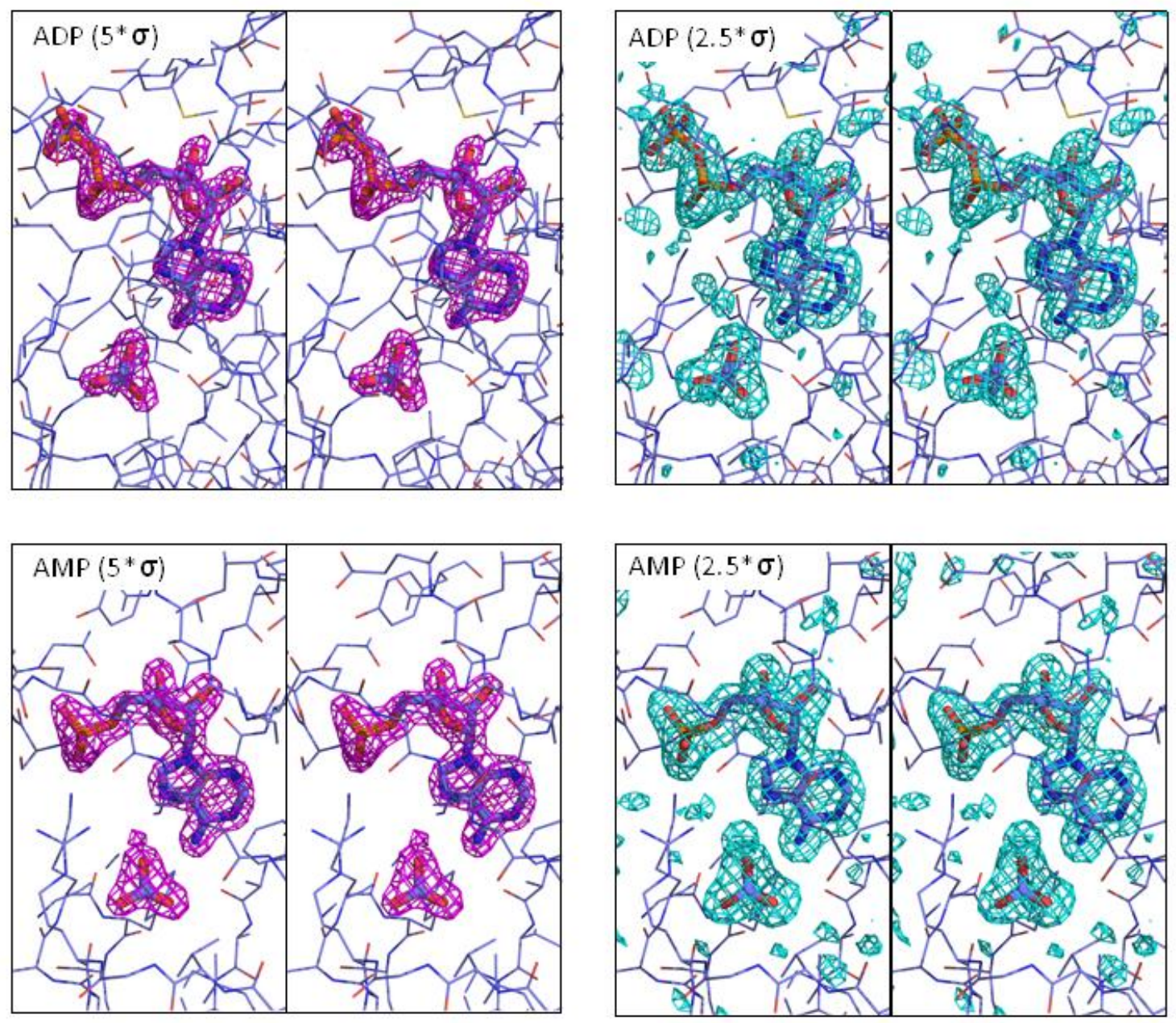

FIGURE 8 
Table 1. X-ray Diffraction Data and Atomic Refinement Statistics

\begin{tabular}{|c|c|c|c|c|c|c|}
\hline PDB ID & 5D4L & 5D4N & 5D40 & 5DRK & 5DS7 & 5D4P \\
\hline Space group & P3 & $\mathrm{P} 2{ }_{1} 2_{1} 2_{1}$ & $\mathrm{P} 2{ }_{1} 2_{1} 2_{1}$ & $\mathrm{P} 2{ }_{1} 2_{1} 2_{1}$ & $\mathrm{P} 2{ }_{1} 2_{1} 2_{1}$ & $\mathrm{P} 2{ }_{1} 2_{1} 2_{1}$ \\
\hline Unit cell: a b c $(\AA ̊)$ & $82.7,82.7,31.5$ & $51.5,75.4,79.6$ & $51.4,75.5,80.0$ & $51.9,75.7,80.3$ & $51.5,75.6,79.9$ & $49.8,76.2,87.9$ \\
\hline Unit cell: $\alpha, \beta, \gamma\left({ }^{\circ}\right)$ & $90,90,120$ & $90,90,90$ & $90,90,90$ & $90,90,90$ & $90,90,90$ & $90,90,90$ \\
\hline Resolution $(\AA ̊)$ & $71.6-2.3$ & $54.7-1.6$ & $54.9-1.8$ & $55.0-2.0$ & $54.9-2.3$ & $43.3-2.2$ \\
\hline Data completeness (\%) & $97.4(91.9)$ & $99.7(97.5)$ & $99.3(94.1)$ & $99.70(96.1)$ & $99.7(94.6)$ & $97.6(91.9)$ \\
\hline Total reflections & $121103(7666)$ & 267556 (19026) & $191688(11606)$ & 160664 (10357) & 160664 (10357) & 98900 (6227) \\
\hline Unique reflections & $10491(747)$ & $41577(2984)$ & 29590 (2037) & 13077 (910) & 13077 (910) & $17217(1177)$ \\
\hline Multiplicity & $11.5(10.3)$ & $6.4(6.4)$ & $6.5(5.7)$ & $14.2(13.1)$ & $12.3(11.4)$ & $5.7(5.3)$ \\
\hline Rmerge & 0.13 & 0.04 & 0.05 & 0.11 & 0.09 & 0.08 \\
\hline $\mathrm{I} / \sigma(\mathrm{I})$ & $17.0(3.2)$ & $21.4(1.9)$ & $19.2(2.7)$ & $22.8(3.5)$ & $20.5(3.1)$ & $15.0(3.0)$ \\
\hline Refinement program & PHENIX & BUSTER-TNT & PHENIX & PHENIX & PHENIX & PHENIX \\
\hline$R$, R-free & $0.177,0.234$ & $0.211,0.234$ & $0.192,0.229$ & $0.230,0.256$ & $0.2439,0.245$ & $0.191,0.247$ \\
\hline $\mathrm{R}$-free test set (reflections) & 525, or $5.3 \%$ & 2095 , or $5.3 \%$ & 2959 , or $11.1 \%$ & 1068 , or $5.0 \%$ & 694 , or $5.0 \%$ & 1720 , or $11.1 \%$ \\
\hline Bonds $(\AA ̊)$ r.m.s.d. from ideal & 0.008 & 0.010 & 0.007 & 0.013 & 0.018 & 0.008 \\
\hline Angles $\left({ }^{\circ}\right)$ r.m.s.d. from ideal & 1.23 & 1.11 & 1.26 & 1.64 & 1.72 & 1.28 \\
\hline \multicolumn{7}{|l|}{ Non-hydrogen atoms } \\
\hline Protein chains/atoms & 2/1474 & $3 / 2242$ & 3/2305 & $3 / 2128$ & $3 / 2198$ & $3 / 2341$ \\
\hline ADP molecules & 0 & 1 & 1 & 1 & 0 & 3 \\
\hline AMP molecules & 0 & 1 & 1 & 1 & 3 & 0 \\
\hline Bicarbonate molecules & 0 & 0 & 2 & 1 & 0 & $7^{*}$ \\
\hline Acetate molecules & 0 & 2 & 0 & 0 & 0 & 0 \\
\hline Waters molecules & 22 & 103 & 100 & 7 & 63 & 45 \\
\hline Number of residues & 194 & 298 & 302 & 284 & 291 & 312 \\
\hline Wilson B-factor $\left(\AA^{2}\right)$ & 28.9 & 30.0 & 28.0 & 37.4 & 27.5 & 34.2 \\
\hline Ave. B-factor $\left(\AA^{2}\right)$ & 34.0 & 35.6 & 33.0 & 44.8 & 33.9 & 43.0 \\
\hline Ramachandran (\% favored) & $184(97.9 \%)$ & $276(100 \%)$ & $287(99.0 \%)$ & $263(98.1 \%)$ & $276(98.9 \%)$ & $300(98.7 \%)$ \\
\hline Ramachandran (\% outliers) & $1(0.5 \%)$ & $0(0 \%)$ & $0(0 \%)$ & $1(0.4 \%)$ & $0(0 \%)$ & $0(0 \%)$ \\
\hline
\end{tabular}


Statistics for the highest-resolution shell are shown in parentheses.

* Only three of the bicarbonates occupy positions believed to be biologically relevant (i.e. in the protein binding cleft). Others appear to represent fortuitous binding within the context of the crystal state. 

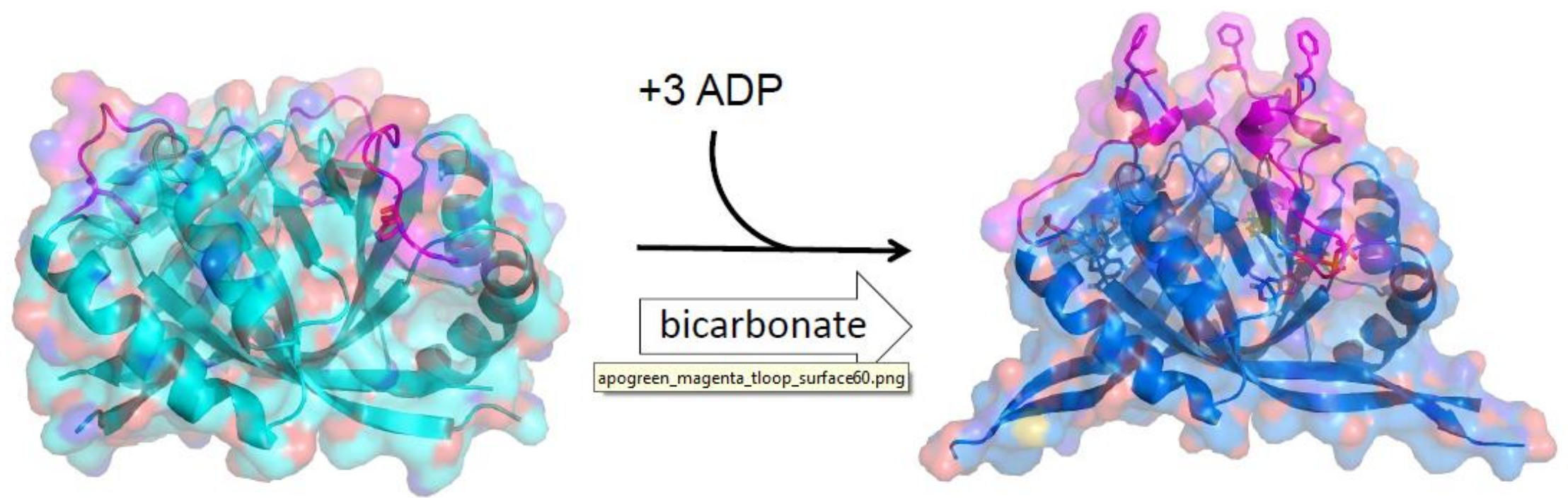

Graphical abstract 